\title{
Vortex suppression and drag reduction in the wake of counter-rotating cylinders
}

\author{
ANDRE S. CHAN ${ }^{1,2} \dagger$, PETER A. DEWEY \\ ANTONY JAMESON ${ }^{1}$, CHUNLEI LIANG G \\ AND ALEXANDER J. SMITS ${ }^{3}$ \\ ${ }^{1}$ Department of Aeronautics and Astronautics, Stanford University, Stanford, CA 94305, USA \\ ${ }^{2}$ Department of Mechanical Engineering, Stanford University, Stanford, CA 94305, USA \\ ${ }^{3}$ Department of Mechanical and Aerospace Engineering, Princeton University, Princeton, NJ 08544, USA
}

(Received 21 July 2010; revised 9 December 2010; accepted 13 March 2011;

first published online 12 May 2011)

The flow over a pair of counter-rotating cylinders is investigated numerically and experimentally. It is demonstrated that it is possible to suppress unsteady vortex shedding for gap sizes from one to five cylinder diameters, at Reynolds numbers from 100 to 200, expanding on the more limited work by Chan \& Jameson (Intl J. Numer. Meth. Fluids, vol. 63, 2010, p. 22). The degree of unsteady wake suppression is proportional to the speed and the direction of rotation, and there is a critical rotation rate where a complete suppression of flow unsteadiness can be achieved. In the doublet-like configuration at higher rotational speeds, a virtual elliptic body that resembles a potential doublet is formed, and the drag is reduced to zero. The shape of the elliptic body primarily depends on the gap between the two cylinders and the speed of rotation. Prior to the formation of the elliptic body, a second instability region is observed, similar to that seen in studies of single rotating cylinders. It is also shown that the unsteady wake suppression can be achieved by rotating each cylinder in the opposite direction, that is, in a reverse doublet-like configuration. This tends to minimize the wake interaction of the cylinder pair and the second instability does not make an appearance over the range of speeds investigated here.

Key words: drag reduction, instability control, vortex interactions

\section{Introduction}

The flow over a pair of non-rotating identical circular cylinders is characterized by the interaction between the cylinder wakes that leads to a number of particular flow states according to the Reynolds number, $R e=U d / v$, and the normalized gap spacing, $g^{*}=g / d$. Here, $U$ is the free stream velocity, $d$ is the cylinder diameter, $v$ is the kinematic viscosity and $g$ is the distance between the two cylinder surfaces. In the Reynolds number range of 100-200, an unsteady two-dimensional vortex

$\dagger$ Present address: Hitachi Global Storage Technologies, San Jose, CA 95119, USA.

$\mp$ Email address for correspondence: jameson@ baboon.stanford.edu

I Present address: Department of Mechanical and Aerospace Engineering, George Washington University, Washington, DC 20052, USA. 
(a)

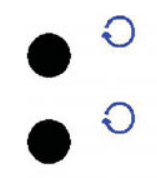

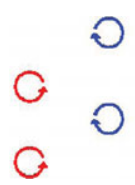

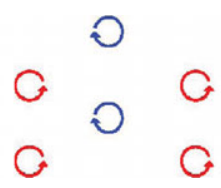

(b)

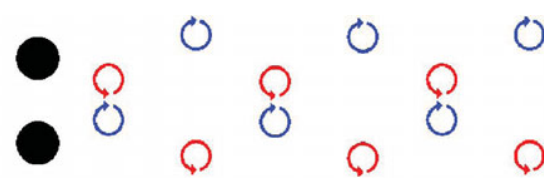

FIGURE 1. (Colour online available at journals.cambridge.org/FLM) Idealized synchronized vortex shedding modes in the wake of a non-rotating cylinder pair. Flow is from left to right. (a) In-phase shedding. (b) Anti-phase shedding.

(a)

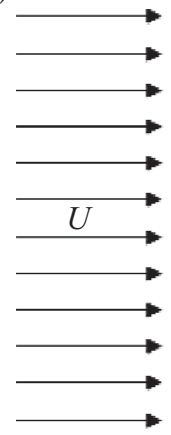

(b)

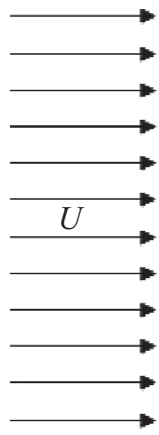

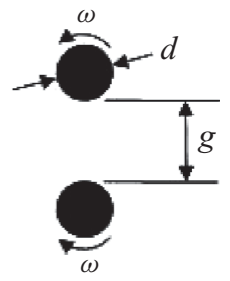

FIGURE 2. Counter-rotating circular cylinder pair. (a) Doublet-like configuration. (b) Reverse doublet-like configuration.

wake has been examined experimentally by Williamson (1985) and $\mathrm{Xu}$, Zhou \& So (2003), numerically by Kang (2003) and analytically by Peschard \& Le Gal (1996). These studies found that for $g^{*}<1$, vortices are shed asynchronously from the cylinder pair, and Kang classified the vortex shedding mode as being either flipflopping $\left(0.4<g^{*}<1.5\right)$ or following a von Kármán vortex street representative of a single bluff body $\left(g^{*}<0.4\right)$. These findings are supported by the work of Kim \& Durbin (1988) and Sumner et al. (1999). For $g^{*} \geqslant 1$, two synchronized vortex shedding modes from the cylinder pair are observed, either in-phase or anti-phase (figure 1). Williamson (1985) noted that for $1<g^{*}<5$ the anti-phase mode is predominant and stable since each vortex generally keeps its form while being gradually dissipated further downstream. Within this gap spacing regime, however, it is possible for the wake to 'flip' to in-phase shedding synchronization and vice versa, particularly for smaller gaps such as $g^{*}=1$. The in-phase mode is unstable as the opposite-signed vortices tend to coalesce into a larger vortex cell. This mode often leads to nonsinusoidal lift and drag variations, and it breaks down into alternative shedding modes when the gap is small. Numerically, the formation of the in-phase shedding mode can be induced by disturbing the flow using either a tripping technique at the beginning of a time marching routine, or by using an artificially large time step and/or introducing some non-streamwise flow component.

When the two cylinders are put into counter-rotation, several recent numerical studies have shown that these wake instabilities can be attenuated and even entirely suppressed (Yoon et al. 2007; Chan \& Jameson 2010). Counter-rotation can be achieved in two different rotational configurations: the doublet-like and reverse doublet-like configuration, as shown in figure 2. Yoon et al. (2007) investigated the doublet-like configuration for a number of gap distances at $R e=100$, and showed that there exists a critical rotational speed, $\Omega_{\text {crit }}$, where the unsteady vortex wakes are completely suppressed. Here $\Omega=\omega /(2 U / d)$, where $\omega$ is the rotational speed of 
the cylinders. Also, decreasing the gap spacing reduces the critical vortex suppression speed. The wake structure present when the cylinders were rotated at speeds below the critical value can be classified in a manner similar to non-rotating cylinders and it depends on both $g^{*}$ and $\Omega$. The unsteady regime at $g^{*}=0.7$ produced a single vortex street, while a larger gap spacing yielded either in-phase or anti-phase synchronous vortex shedding. Chan \& Jameson (2010) extended this work by showing that the unsteady vortex wake could be suppressed in the reverse doublet-like configuration as well. For $R e=150$ and $g^{*}=1$, they show that $\Omega_{c r i t}=1.5$ for the doublet-like configuration and 3.5 for the reverse doublet-like configuration. Additionally, they found that for the doublet-like configuration, an increase in rotational speed past the critical speed created a virtual elliptic body that resembled a doublet potential flow.

Here, we examine the flow past two counter-rotating cylinders in more detail, where the cylinders rotate at the same rate. We refine the previous numerically obtained results using a higher order simulation method, and confirm the computations using physical experiments. In the work by Chan \& Jameson (2010), the simulations were performed with a commercial computational fluid dynamics (CFD) code for incompressible flow simulations, CFD-ACE+, from the ESI Corporation. In the current work, we use a high-order spectral difference (SD) method developed recently for compressible flow simulations. The accompanying experiments were performed in a water channel using digital particle image velocimetry. We focus on relatively low Reynolds numbers so that we can assume that the flow past the counterrotating circular cylinder is predominantly two-dimensional. We use these techniques to investigate the effect on the wake structure of gap spacing $\left(g^{*}=1,3,5\right)$, Reynolds number $(R e=100,150,200)$ and rotational speed $(0 \leqslant \Omega \leqslant 5)$, focusing on the unsteady suppression for the doublet-like and reverse doublet-like configurations, and on the formation of virtual elliptical bodies. In addition, we describe for the first time the presence of a second region of instability at rotation rates above the critical value. A particular highlight of the present work is the remarkable agreement found between the numerical and experimental results.

Our study was inspired by the original work of Prandtl \& Tietjens (1934) who hypothesized that steady, symmetric streamlines can be generated by two touching cylinders rotating in the opposite direction. The motivation also comes from Prandtl's subsequent experimental work on a single rotating cylinder which led to the design of the Flettner rotor ship. We expect that the concept of the virtual elliptic body may find applications in ocean and wind engineering (see, for example, Whittlesey, Liska \& Dabiri 2010).

\section{Numerical methods}

\subsection{Introduction}

While second-order methods for computational simulations of fluid flow are by now mature and reliable, they introduce levels of numerical diffusion that cause rapid attenuation of concentrated regions of vorticity. Consequently, accurate simulation of vortex-dominated flows can most readily be achieved by resorting to higher order methods. One of the most promising approaches is the discontinuous Galerkin (DG) method, for which the theoretical basis has been provided by Cockburn \& Shu (1989), Cockburn, Lin \& Shu (1989), Cockburn, Hou \& Shu (1990) and Cockburn \& Shu (1998). The rapid growth of the computational complexity of DG methods with increasing order has spurred the search for more efficient variants or alternatives. One approach is the nodal DG scheme in which the solution is represented by Lagrange interpolation at a set of collocation points in each element, and the quadratures 
required by the DG method are pre-integrated to produce local mass and stiffness matrices (Hesthaven \& Warburton 2008).

The spectral difference (SD) method has recently emerged as a promising alternative. The basic concept of the SD method was first put forward by Kopriva \& Kolias (1996) under the name 'staggered grid Chebyshev multidomain' method. In order to discretize the conservation law

$$
\frac{\partial u}{\partial t}+\frac{\partial}{\partial x} f(u)=0
$$

they proposed to represent the solution by polynomials of degree $p$ in each element and the flux by polynomials of degree $p+1$, with interlocking collocation points for the solution and the flux. The flux collocation points include the element boundaries, where a single-valued numerical flux is imposed which is common to each element and its neighbours on the left or right. Then, the value of $\partial u / \partial t$ at each solution point is obtained directly as the derivative of the flux polynomial. Kopriva \& Kolias (1996) used Chebyshev and Chebyshev-Lobatto points as the solution and flux collocation points, and it remains unclear whether the SD scheme is stable with this choice, although they did prove the scheme to be conservative. Some years later, Liu, Vinokur \& Wang (2006) presented a general formulation of SD methods on both quadrilateral and triangular elements. While the SD method has proved robust and productive in a variety of applications (Wang et al. 2007; Mohammad, Wang \& Liang 2008; Ou et al. 2009; Premasuthan et al. 2009; Liang, Jameson \& Wang 2009a; Liang, Premasuthan \& Jameson 2009b; Liang et al. 2009c), doubts persist about its stability. For example, Huynh (2007) showed that it can be weakly unstable in one dimension depending on the choice of flux collocation points, including the choice made by Kopriva \& Kolias (1996). In contrast, Jameson (2010) proved that it is stable in an energy norm for all orders of accuracy if the interior flux collocation points are chosen as the zeros of the corresponding Legendre polynomials.

The implementation of the SD method on quadrilateral or hexahedral elements is simple as it allows one to use polynomial interpolation based on tensor product forms rather than the multivariate interpolation used with triangular or tetrahedral elements. The formulation is briefly reviewed in the next section.

\subsection{SD spatial discretization in two dimensions}

Consider the unsteady compressible 2D Navier-Stokes equations in conservative form

$$
\frac{\partial \boldsymbol{Q}}{\partial t}+\frac{\partial \boldsymbol{f}}{\partial x}+\frac{\partial \boldsymbol{g}}{\partial y}=0,
$$

where $\boldsymbol{Q}$ is the vector of conserved variables, and $\boldsymbol{f}$ and $\boldsymbol{g}$ are the total fluxes including both inviscid and viscous flux vectors. The inviscid and viscous fluxes of the Navier-Stokes equations can be written separately in the following form:

$$
\frac{\partial \boldsymbol{Q}}{\partial t}+\nabla \boldsymbol{F}_{e}(\boldsymbol{Q})+\nabla \boldsymbol{F}_{v}(\boldsymbol{Q}, \boldsymbol{\nabla} \boldsymbol{Q})=0,
$$

where the conservative variables $\boldsymbol{Q}$ and Cartesian components $\boldsymbol{f}_{e}(\boldsymbol{Q})$ and $\boldsymbol{g}_{e}(\boldsymbol{Q})$ of the inviscid flux vector $\boldsymbol{F}_{e}(\boldsymbol{Q})$ are given by

$$
\boldsymbol{Q}=\left[\begin{array}{c}
\rho \\
\rho u \\
\rho v \\
E
\end{array}\right], \quad \boldsymbol{f}_{e}(\boldsymbol{Q})=\left[\begin{array}{c}
\rho u \\
\rho u^{2} \\
\rho u v \\
u(E+p)
\end{array}\right], \quad \boldsymbol{g}_{e}(\boldsymbol{Q})=\left[\begin{array}{c}
\rho v \\
\rho u v \\
\rho v^{2}+p \\
v(E+p)
\end{array}\right] .
$$


Here $\rho$ is the density, $u$ and $v$ are the velocity components in the $x$-and $y$-directions, $p$ is the pressure and $E$ is the total energy. The pressure is related to the total energy by

$$
E=\frac{p}{\gamma-1}+\frac{1}{2} \rho\left(u^{2}+v^{2}\right)
$$

with a constant ratio of specific heat $\gamma$. For all numerical test cases in the present study, $\gamma=1.4$ for air.

The Cartesian components $\boldsymbol{f}_{v}(\boldsymbol{Q}, \nabla \boldsymbol{Q})$ and $\boldsymbol{g}_{v}(\boldsymbol{Q}, \nabla \boldsymbol{Q})$ of the viscous flux vector $\boldsymbol{F}_{v}(\boldsymbol{Q}, \nabla \boldsymbol{Q})$ are given by

$$
\begin{gathered}
\boldsymbol{f}_{v}(\boldsymbol{Q}, \nabla \boldsymbol{Q})=\mu\left[\begin{array}{c}
0 \\
2 u_{x}+\lambda\left(u_{x}+v_{y}\right) \\
v_{x}+u_{y} \\
u\left[2 u_{x}+\lambda\left(u_{x}+v_{y}\right)\right]+v\left(v_{x}+u_{y}\right)+\frac{C_{p}}{P r} T_{x}
\end{array}\right], \\
\boldsymbol{g}_{v}(\boldsymbol{Q}, \nabla \boldsymbol{Q})=\mu\left[\begin{array}{c}
0 \\
v_{x}+u_{y} \\
2 v_{y}+\lambda\left(u_{x}+v_{y}\right) \\
v\left[2 v_{y}+\lambda\left(u_{x}+v_{y}\right)\right]+u\left(v_{x}+u_{y}\right)+\frac{C_{p}}{P r} T_{y}
\end{array}\right],
\end{gathered}
$$

where $\mu$ is the dynamic viscosity, $C_{p}$ is the specific heat at constant pressure, $\operatorname{Pr}$ is the Prandtl number and $T$ is the temperature ( $\lambda$ is set to $-2 / 3$ according to the Stokes hypothesis).

To achieve an efficient implementation, all quadrilateral elements in the physical domain $(x, y)$ determined by the one-level $h$-refinement are transformed into a standard square element $(0 \leqslant \xi \leqslant 1,0 \leqslant \eta \leqslant 1)$.

The governing equations in the physical domain are then transferred into the computational domain, and the transformed equations take the following form:

$$
\frac{\partial \tilde{\boldsymbol{Q}}}{\partial t}+\frac{\partial \tilde{\boldsymbol{f}}}{\partial \xi}+\frac{\partial \tilde{\boldsymbol{g}}}{\partial \eta}=0
$$

where

$$
\tilde{\boldsymbol{Q}}=|\boldsymbol{J}| \cdot \boldsymbol{Q}, \quad \boldsymbol{J}=\left(\begin{array}{ll}
x_{\xi} & x_{\eta} \\
y_{\xi} & y_{\eta}
\end{array}\right) \quad \text { and } \quad\left(\begin{array}{c}
\tilde{\boldsymbol{f}} \\
\tilde{\boldsymbol{g}}
\end{array}\right)=|\boldsymbol{J}| \boldsymbol{J}^{-1}\left(\begin{array}{c}
\boldsymbol{f} \\
\boldsymbol{g}
\end{array}\right) .
$$

In the standard element, two sets of points are defined, namely the solution points and the flux points. A fourth-order representation is illustrated as an example in figure 3 .

The solution is represented as a tensor product of polynomials of degree $N-1$ using a Lagrange basis defined as

$$
h_{i}(X)=\prod_{s=0, s \neq i}^{N}\left(\frac{X-X_{s}}{X_{i}-X_{s}}\right)
$$

at $N$ solution points $X_{i}$. The flux along a line of solution points is represented by a polynomial of degree $N$ using a staggered basis including points at the element interface:

$$
l_{i+1 / 2}(X)=\prod_{s=0, s \neq i}^{N}\left(\frac{X-X_{s+1 / 2}}{X_{i+1 / 2}-X_{s+1 / 2}}\right) .
$$




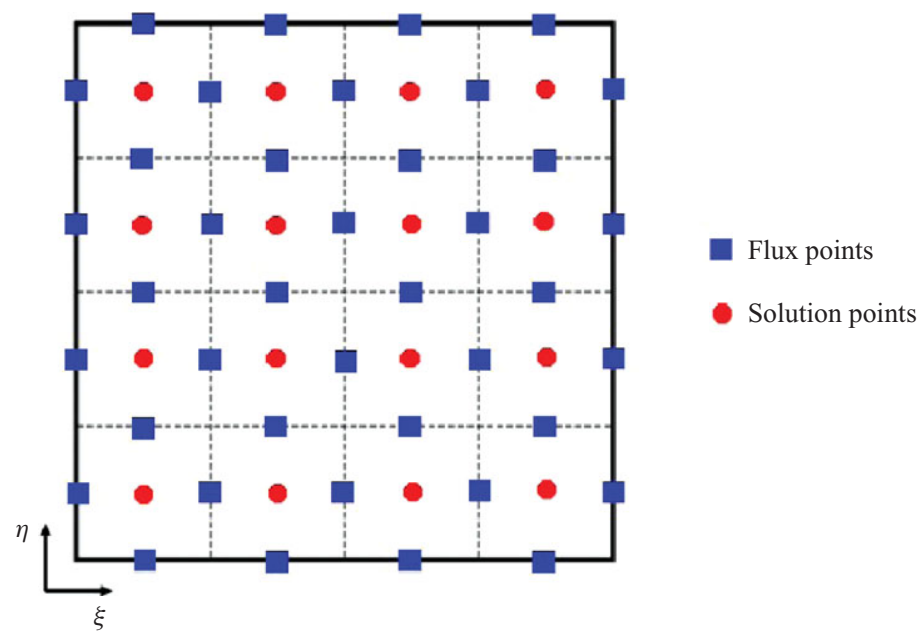

Figure 3. (Colour online) Distribution of flux and solution points for the fourth-order SD scheme.

The reconstructed solution for the conserved variables takes the form

$$
\boldsymbol{Q}(\xi, \eta)=\sum_{j=1}^{N} \sum_{i=1}^{N} \frac{\tilde{\boldsymbol{Q}}_{i, j}}{\left|J_{i, j}\right|} h_{i}(\xi) \cdot h_{j}(\eta) .
$$

Similarly, the reconstructed flux polynomials take the following form:

$$
\begin{aligned}
\tilde{\boldsymbol{f}}(\xi, \eta) & =\sum_{j=1}^{N} \sum_{i=0}^{N} \tilde{\boldsymbol{f}}_{i+1 / 2, j} l_{i+1 / 2}(\xi) \cdot h_{j}(\eta) \\
\tilde{\boldsymbol{g}}(\xi, \eta) & =\sum_{j=1}^{N} \sum_{i=0}^{N} \tilde{\boldsymbol{g}}_{i, j+1 / 2} h_{i}(\xi) \cdot l_{j+1 / 2}(\eta) .
\end{aligned}
$$

Thus $\partial \tilde{\boldsymbol{f}} / \partial \xi$ and $\partial \tilde{\boldsymbol{g}} / \partial \eta$ reduce to polynomials of degree $N-1$ consistent with the solution. Following the analysis of Huynh (2007) and Jameson (2010), the flux points are selected as the Legendre-Gauss quadrature points plus the two end points, 0 and 1. The locations of the interior Legendre-Gauss quadrature points are the roots of the equation $P_{n}(\xi)=0$.

For the inviscid flux, a Riemann solver using the Roe (1981) scheme is employed to compute a common flux at interfaces to ensure conservation and stability. In the present study, the flux vector at the element boundary interfaces is computed with the entropy fix suggested by Harten \& Hyman (1983). The viscous fluxes are computed from the average of the flux point gradients on the element interfaces as outlined by Liang et al. (2009a). All computations presented here are advanced in time using a fourth-order strong-stability-preserving five-stage Runge-Kutta scheme (Spiteri \& Ruuth 2002).

The SD code used in this research has been extensively validated and documented by Liang et al. (2009b) and Ou et al. (2009), and it has been verified that the order of accuracy is $N$ when using $N \times N$ solution points in each element. Additional code validation tests are given in the Appendix. 
(a)

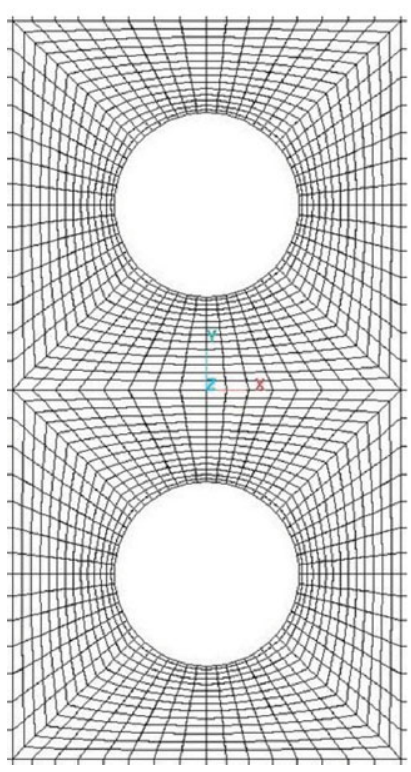

(b)

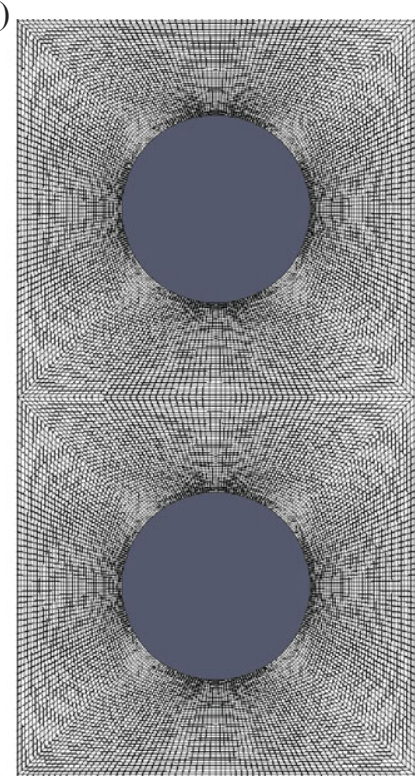

FIGURE 4. (Colour online) ( $a$ ) Mesh around the cylinder pair surfaces $\left(g^{*}=1\right)$. (b) The fourth-order SD solution collocation points around the cylinder pair surfaces $\left(g^{*}=1\right)$.

\subsection{Model set-up}

In order to perform the SD simulation, we start by constructing a computational domain using a commercial mesh generator. The size of the computational domain is $66.328 d \times 100 d$ consisting of 5176, 7736 and 9088 cells for $g^{*}=1,3$ and 5, respectively. Simulations were performed at Reynolds numbers of 100, 150 and 200. The mesh is constructed to be finer near the cylinder surface (figure 4) and progressively coarser radially outward. Figure 4 illustrates the solution collocation points (16 per cell) by the fourth-order SD around a cylinder surface. The computational domains for other values of $g^{*}$ are constructed in a similar manner. Our study, as shown in $\S$ A.3 of the Appendix, indicates that the fourth-order SD solutions using these mesh densities lead to mesh-independent results with sufficient accuracy, which is consistent with $\mathrm{Ou}$ et al. (2009).

Across the inlet, the Dirichlet boundary condition is specified with a uniform velocity of $0.1 \mathrm{~m} \mathrm{~s}^{-1}$. The position of the inlet plane is far enough upstream for the flow to be considered unbounded and to have negligible influence on the vortex shedding behaviour of the cylinder pair. The upper and lower boundary conditions are set to a symmetrical slip condition. At the exit boundary, a fixed pressure equal to the free stream condition is specified while other flow variables are extrapolated so that the flow remains uniform at the inlet. An isothermal, no slip boundary condition is applied on the cylinder wall surfaces.

One of the main advantages of using the SD scheme is that we can reduce the total computational time by first producing a less accurate result using a lower order scheme (that is, second order) to establish the general flow pattern and then switching to higher order (that is, fourth order) after the error residual norm has converged to a satisfactory level. In addition, any solution from a higher order scheme can be used to restart another solution of a lower scheme. This proved to be particularly useful when stepping through different rotational speeds. 

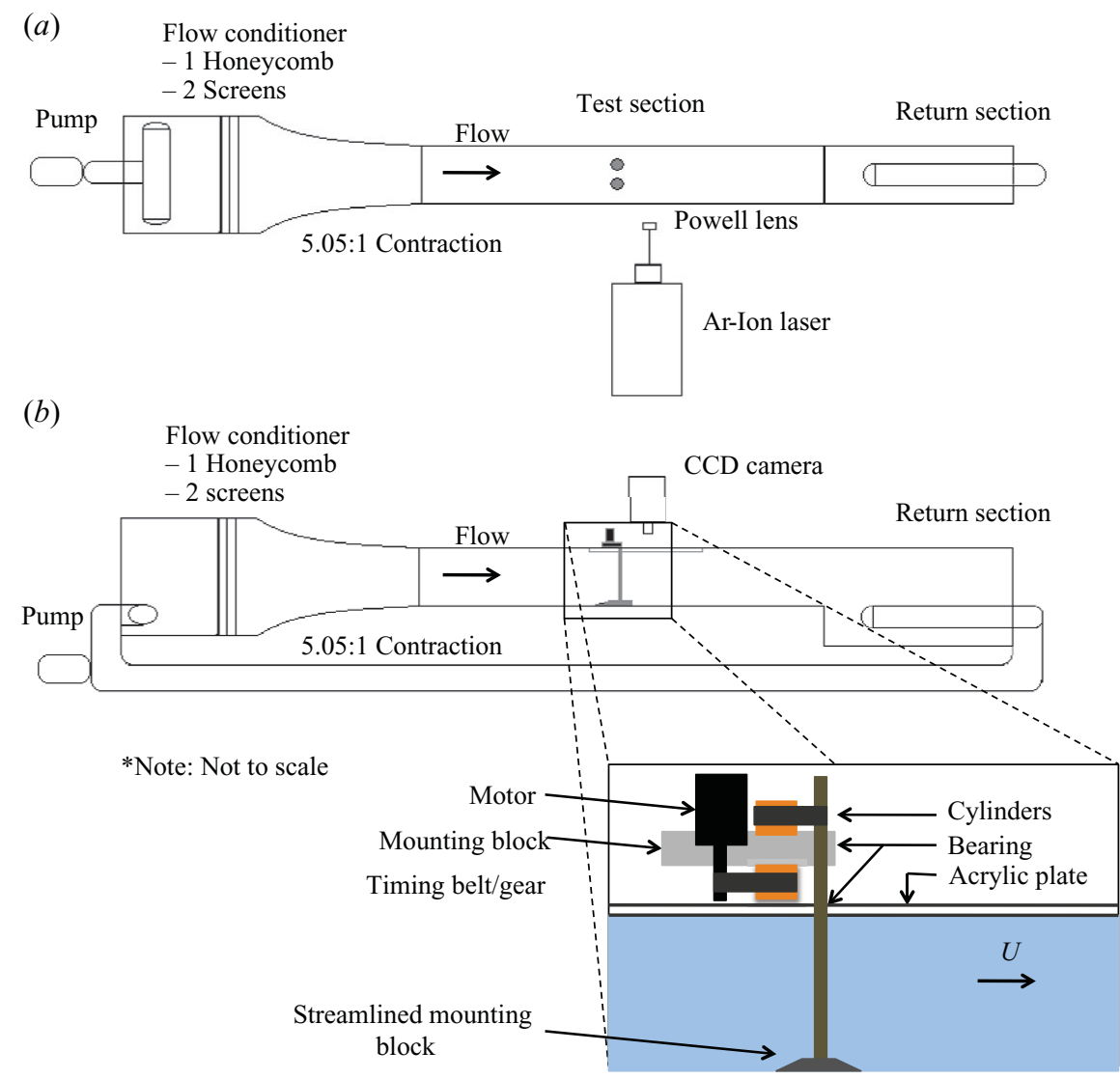

FIGURE 5. (Colour online) Sketch of water channel and experimental apparatus. ( $a$ ) Top view; $(b)$ side view. Note: drawings not to scale.

We consider steady and unsteady flow solutions. The results shown in the next section are determined when the computation reaches a statistically stable solution and from which the solution from the impulsive start is excluded. Since we primarily focus on the suppression of the unsteady vortex shedding, we are less interested in how the final solution is established. Hence, in order to balance the solution accuracy and the computational time, the fourth-order SD method is only implemented to obtain the final flow solution.

\section{Experimental methods}

The flow past the counter-rotating cylinder pair was investigated experimentally using digital particle image velocimetry (DPIV). To achieve counter-rotation of a cylinder pair, a Pittman DC motor (9236S009-R1) attached to a timing belt with a $6: 1$ gear reduction ratio was used to rotate one of the cylinders, and the second cylinder was coupled to the first via a spur gear to produce counter-rotation at the same rate. The rotation speed was determined by sampling the output of a digital optical encoder accompanying the motor at a frequency of $500 \mathrm{~Hz}$. The apparatus was mounted in a closed-loop, free surface water channel having a test section 0.46 $\mathrm{m}$ wide, $0.3 \mathrm{~m}$ deep and $2.5 \mathrm{~m}$ long (see figure 5) with free stream turbulence intensity less than $4 \%$. Surface waves were eliminated by mounting a clear acrylic plate; 
$0.0127 \mathrm{~m}$ thick, $0.45 \mathrm{~m}$ wide and extending $0.61 \mathrm{~m}$ upstream and downstream of the cylinders, to be in contact with the free surface, with the cylinder pair mounted vertically and protruding through a slot machined in the acrylic plate. The bottoms of the cylinders were held in place by low-friction bearings, housed in a streamlined mounting block that extended $10 \mathrm{~mm}$ above the channel floor and had a foot-print of $0.066 \mathrm{~m} \times 0.03 \mathrm{~m}$. There were no additional end plates on the cylinders. The cylinders were $0.00635 \mathrm{~m}$ in diameter and $0.3 \mathrm{~m}$ long, producing an aspect ratio of 47 . The DPIV results were used to examine the two-dimensional continuity equation in three different spanwise planes along the cylinder pair (midspan and midspan $\pm 10 d$ ) to verify that three-dimensional effects were negligible. All other data were acquired at the midspan of the cylinders.

Neutrally buoyant silver-coated hollow ceramic spheres with a mean diameter of $93 \mu \mathrm{m}$ were used as seeding particles (Potters Industries Inc. Conduct-O-Fil AGSL150 TRD). A Spectra Physics 2020 Argon-Ion laser coupled to a fibre optic delivery system and Powell lens (Oz Optics Ltd.) were used to produce a 1.5-mm-thick light sheet for the experiments. A Redlake MotionXtra HG-LE CCD camera with $1128 \times 752$ pixel resolution was used to acquire the DPIV images. The camera frame rates ranged between 50 and $100 \mathrm{~Hz}$, producing 30-60 discrete time intervals between subsequent vortices being shed, with a typical exposure time of $3 \mathrm{~ms}$.

Two-dimensional discretely sampled velocity fields were computed by calculating local spatial cross-correlations using software developed by Jiménez (2002). Three passes, with $64 \times 64,32 \times 32$, and subsequently $16 \times 16$ pixel windows with $50 \%$ overlap were performed. To achieve sub-pixel accuracy, a $5 \times 5$ pixel Gaussian interpolation was utilized. In a typical DPIV pair, vector outliers were primarily constrained to the edges of the domain and regions of the domain with insufficient lighting (for instance, in the shadows created by the cylinders). Fewer than $1 \%$ of vectors in the far-wake of the cylinders proved to be outliers, though resolving the near-wake of the cylinders was more challenging due to lighting issues. The analysis yielded, at most, $5 \%$ error in velocity and $10 \%$ error in vorticity. The vorticity was calculated at each point by computing the circulation about a closed contour formed by the eight neighbouring points using a trapezoidal rule scheme (Raffel et al. 1998). There is an estimated error of $9 \%$ in the determination of the critical rotation speed to suppress vortex formation, $\Omega_{\text {crit }}$.

The DPIV system was validated by examining the flow past a stationary cylinder. A fast Fourier transform (FFT) was taken of the time-resolved velocity field in the cylinder wake to determine the vortex shedding frequency $f$, and ultimately the Strouhal number, $S t=f d / U$. The Strouhal number was found to be $0.163,0.184$ and 0.197 for Reynolds numbers of 100,150 and 200, respectively, deviating less than $0.05 \%$ from Williamson (1989), see the Appendix for further detail. There is an estimated uncertainty of $5 \%$ in the calculation of the Strouhal number. Note that the shedding frequency was captured equally well by taking the FFT of the vorticity field. The Strouhal number was found to be invariant for three different DPIV spatial resolutions $(2.5,5$ and 8.5 vectors per cylinder diameter), and a spatial resolution of 5 vectors per cylinder diameter was selected for all results presented here.

A typical data set consisted of 1200 image pairs that were phase averaged at the vortex shedding frequency to minimize noise. The phase-averaging was a two-step process: a first data set was used to find the shedding frequency from the cylinder pair, and then a second data set was acquired with the camera timing synchronized with the vortex shedding frequency. It should be noted that the time-resolved data were always used to determine quantities such as $\Omega_{\text {crit }}$ and Strouhal number, and the 
phase-averaged data were solely used for qualitative purposes in displaying vorticity contour plots. In the case where counter-rotation of the cylinder pair suppressed unsteadiness, all image pairs of the original data set were used in the averaging process. Due to optical constraints, only the wake region of the cylinder pair was investigated.

\section{Results}

\subsection{Doublet-like counter-rotating cylinder pair}

Here, we present results for the doublet-like counter-rotating cylinder pair (see figure 2) with $g^{*}=1,3$ and 5, and $R e=100,150$ and 200. In this configuration, the cylinders rotate in a manner that enhances the interaction of the vortex streets in the wake of each individual cylinder, and there is a critical rotation rate at which the wake instabilities are suppressed completely. Increasing the gap spacing corresponds to an attenuation in the interaction, corresponding to a higher critical rotational speed. Nonetheless, relatively low rotational speeds $\left(\Omega_{c r i t}<2\right)$ were required to suppress wake instabilities for all cases.

For $g^{*}=3$ and 5 , the stable anti-phase shedding mode was observed when the cylinders were rotated below $\Omega_{\text {crit }}$ for all Reynolds numbers. The case for $g^{*}=3$ and $R e=200$ is shown in figure 6 . Note the excellent qualitative agreement between the computation and the experiment. In this particular mode, the vortices shed by the cylinder pair remain stable and coherent throughout the entire domain. As the rotational speed increases, the vortex strength decreases until the unsteady vortex wake is entirely suppressed. At a rotational rate at or slightly above $\Omega_{\text {crit }}$, vorticity lobes extend downstream from the surface of the cylinders, but with a further increase in rotational speed the vorticity remains concentrated in the immediate area of the cylinders.

For the cases with $g^{*}=1$, the unstable in-phase vortex shedding mode was observed at $R e=150$ and 200 for all rotational speeds below the critical value. At the lowest Reynolds number investigated, $R e=100$, the vortices shed by the cylinder pair coalesced to form a single von Kármán vortex street, shown in figure 7. This phenomenon was also observed at the same Reynolds number by Yoon et al. (2007) for $g^{*}=0.7$. In the case of a non-rotating cylinder pair, the single vortex street shedding mode is only observed at smaller gap spacings $\left(g^{*}=0.4\right)$, as found by Kang (2003), so that counter-rotation of the cylinders increases the allowable gap spacing to achieve this particular mode. A more detailed discussion of vortex suppression speeds is taken up in $\S 4.3$.

Increasing the rotational speed above $\Omega_{\text {crit }}$ results in a closed vortex system taking the form of a virtual elliptical body, similar in appearance to that of a doublet in potential flow. The computational results clearly display the evolution of this flow pattern, as seen in figure 8. The experimental DPIV data did not have sufficient spatial resolution to resolve the stagnation point created by the closed vortex system. Instead, streaklines in the immediate wake were visualized using 10 consecutive images obtained with a Sony HDR-SR10 HD camcorder recording at 30 f.p.s. These streaklines, shown in figure 9, clearly show the presence of the stagnation point in the wake of the cylinder pair, indicating the presence of the virtual elliptical body above a certain rotational speed. This also confirms the predictions of Chan \& Jameson (2010) made using a lower order numerical simulation. 
(a)
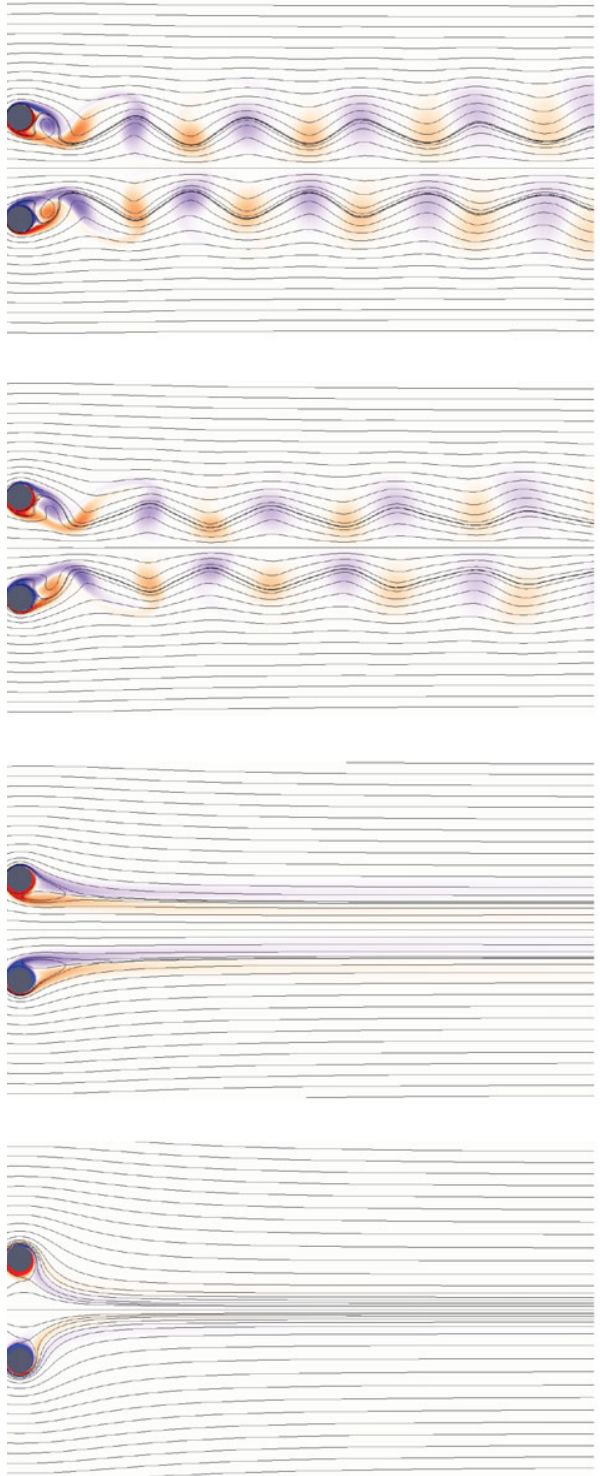

(b)
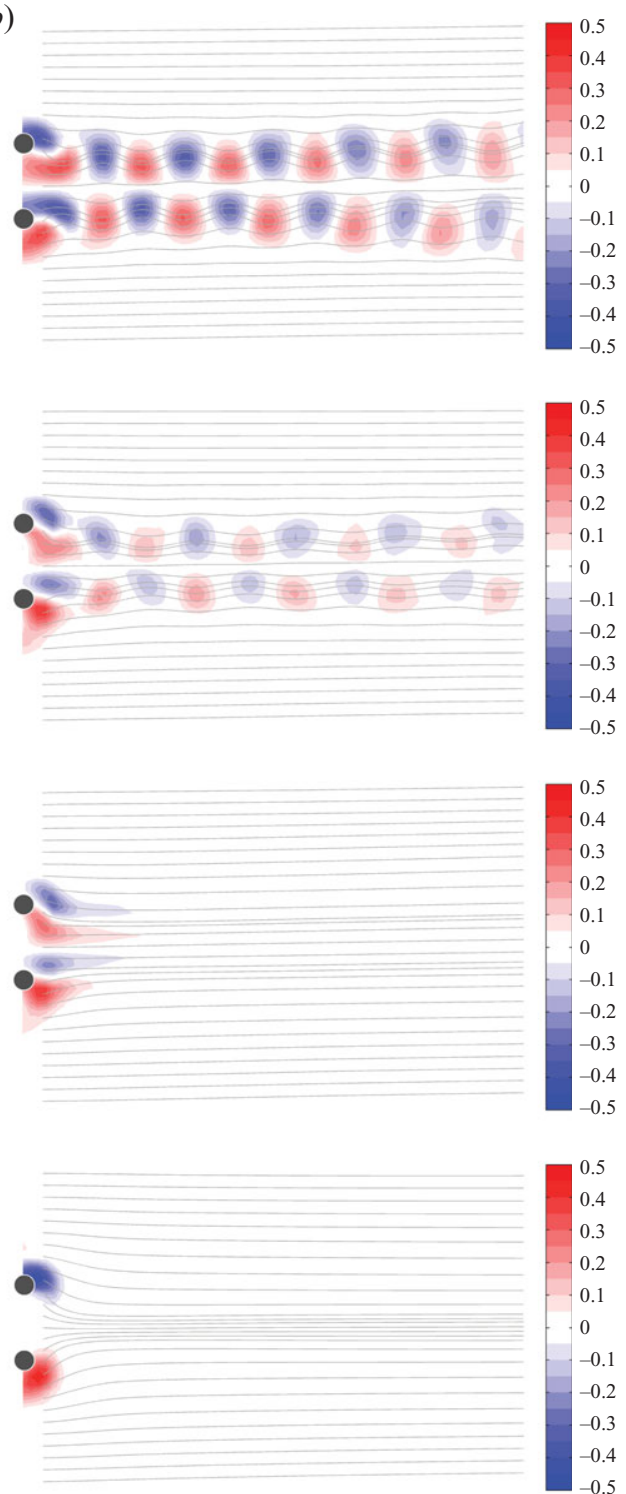

FiguRE 6. Doublet-like rotational configuration: streamlines and vorticity at various rotational speeds for $g^{*}=3, R e=200$. (a) Computational results. (b) Experimental results. First row, $\Omega=1$; second row, $\Omega=1.5$; third row, $\Omega=1.85$ (computational) and 1.88 (experimental); fourth row, $\Omega=3$. For this case, $\Omega_{\text {crit }}=1.85$ (computational) and 1.88 (experimental). Vorticity contour levels for the computational and experimental results are the same.

The lift and drag coefficients are defined by

$$
C_{L}=\frac{L}{\frac{1}{2} \rho U^{2} d}, \quad C_{D}=\frac{D}{\frac{1}{2} \rho U^{2} d},
$$

where $L$ and $D$ are the lift and drag forces that are numerically evaluated by summing the normal and tangential forces from the pressure and viscous stress terms. 
(a)
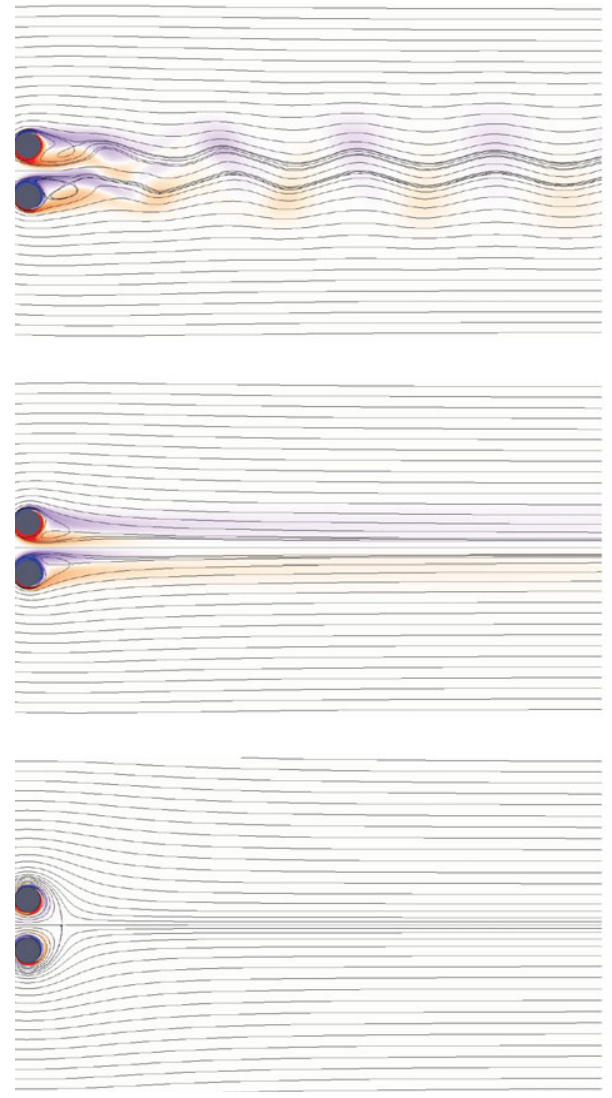

$(b)$
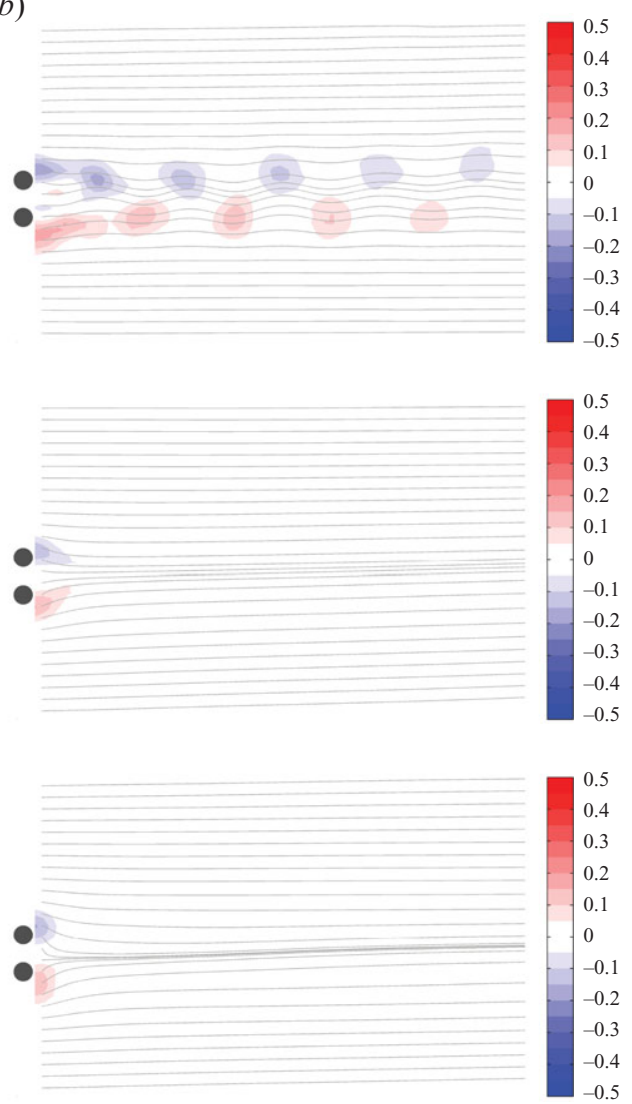

FIGURE 7. Doublet-like rotational configuration: streamlines and vorticity at various rotational speeds for $g^{*}=1, R e=100$. (a) Computational results. (b) Experimental results. First row, $\Omega=1.2$; second row, $\Omega=1.5$; third row $\Omega=3$. For this case, $\Omega_{\text {crit }}=1.35$ (computational) and 1.4 (experimental). Vorticity contour levels for the computational and experimental results are the same.

By rotating a cylinder, positive or negative lift can be generated depending on the direction of the spin. From potential flow theory, the lift force asymptotically increases with spin rate. In a viscous fluid, however, Mittal \& Kumar (2003) showed that at $R e=200$, the potential flow theory over-predicts the lift curve of a single rotating cylinder in the range of $0 \leqslant \Omega \leqslant 5$. The main contribution to the lift in the case of the rotating cylinder pair comes from the pressure term while the viscous stress contribution is relatively small. Figure 10 shows the time-averaged values of the lift coefficient $\overline{C_{L}}$ for the upper cylinder in the doublet-like rotation. Though not shown, $\overline{C_{L}}$ for the lower cylinder has the same amplitude but opposite sign, yielding a net zero lift for the pair, as expected. The results are virtually independent of Reynolds number, and in the lower speed range $(0 \leqslant \Omega \leqslant 2.5)$ they are also independent of gap spacing. The value of $\overline{C_{L}}$ for each cylinder is close to the value obtained by Mittal \& Kumar (2003) at $R e=200$ for a single rotating cylinder. The interaction between the two cylinders prevents the exponential increase of $\overline{C_{L}}$ with rotation rate seen in the case of the single cylinder. The observation that the coefficient of lift does not depend on gap spacing at the lower rotational speeds is directly correlated with the creation of the virtual elliptic body: once the virtual elliptic body appears, $\overline{C_{L}}$ begins 
(a)

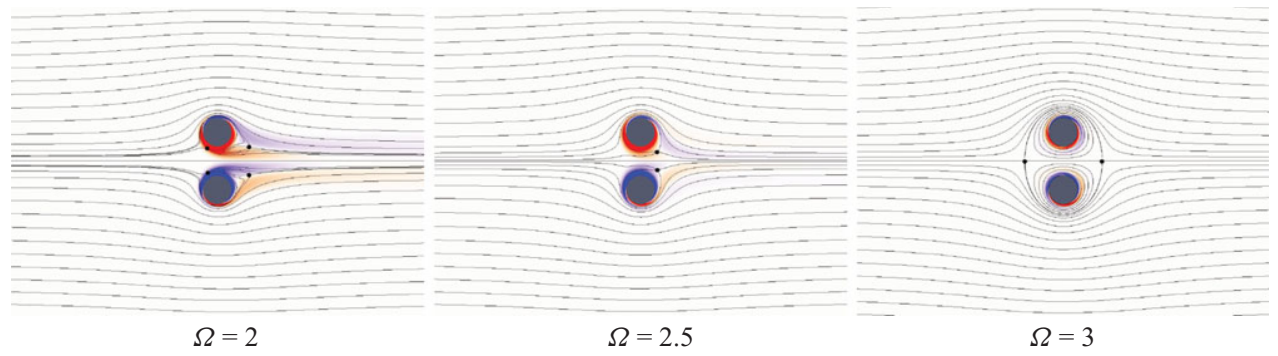

(b)
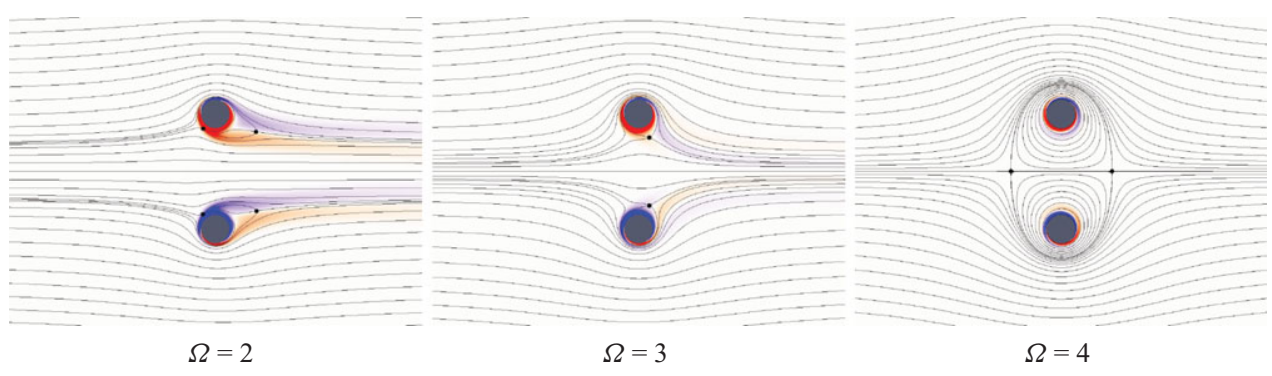

(c)

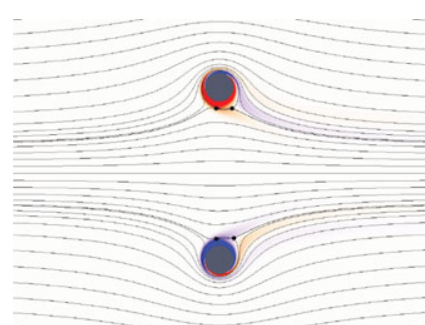

$\Omega=3$

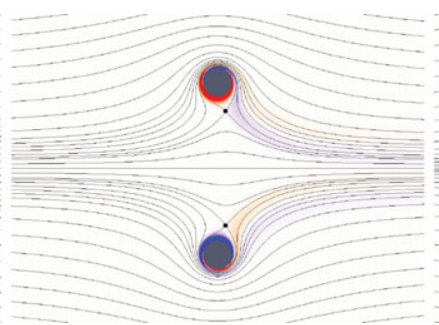

$\Omega=3.5$

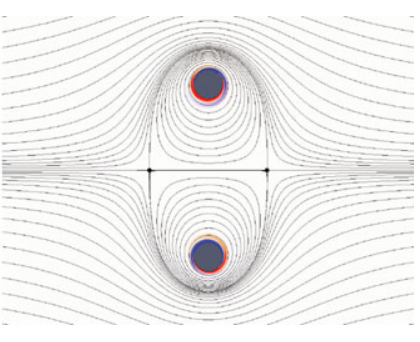

$\Omega=5$

FIGURE 8. Instantaneous streamlines and vorticity showing the evolution of virtual elliptical body for the doublet-like configuration (computational results). (a) $g^{*}=1, \operatorname{Re}=150 ;(b) g^{*}=3$, $R e=150 ;(c) g^{*}=5, R e=150$. Stagnation points are represented by ' $\bullet$ '. Vorticity contour levels are -0.5 (blue) and 0.5 (red).

to depend on gap spacing. As shown in figure 8, the rotational speed to create the virtual elliptic body increases with increasing gap spacing, and it is for this reason that $\overline{C_{L}}$ at $g^{*}=1$ is the first case to deviate from the larger gap spacings, as seen in figure 10. A deviation between $g^{*}=3$ and $g^{*}=5$ does not occur until a rotational speed of $\Omega \approx 4.5$, very close to the rotational speed required to create the virtual elliptic body at $g^{*}=3$.

For most of the investigated values of the Reynolds number and $g^{*}$, the lift forces cause a repelling force on each cylinder. The only exception is for $g^{*}=1$ and $\Omega>4$ where the faster rotation produces an attracting force. The non-monotonic trend of $\overline{C_{L}}$ for $g^{*}=1$, which has a maximum at $\Omega \approx 2.65$, is associated with the creation of the virtual elliptic body. With the creation of a closed streamline surrounding the cylinder pair, the local fluid immediately adjacent to the cylinders becomes increasingly dependent on the cylinder rotation. The flow between the cylinder pair reverses direction and opposes the free stream velocity after the formation of the virtual elliptic body. As the cylinder pair rotational speed is increased, this reversed 
(a)

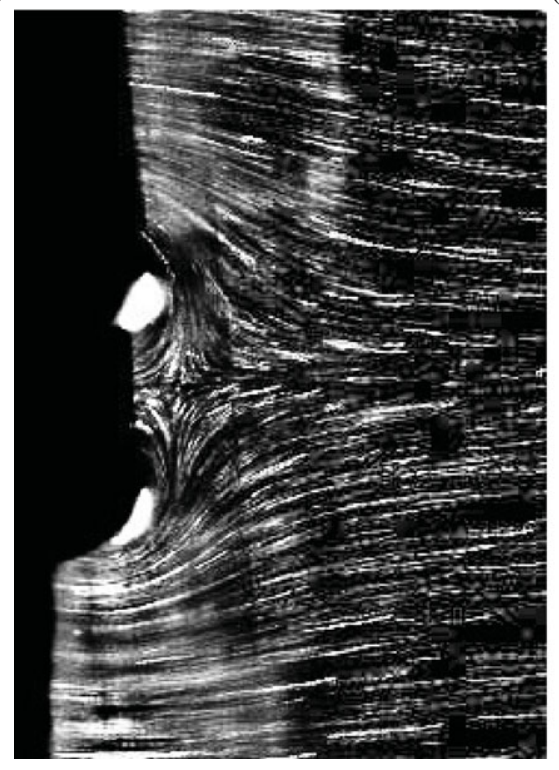

(b)

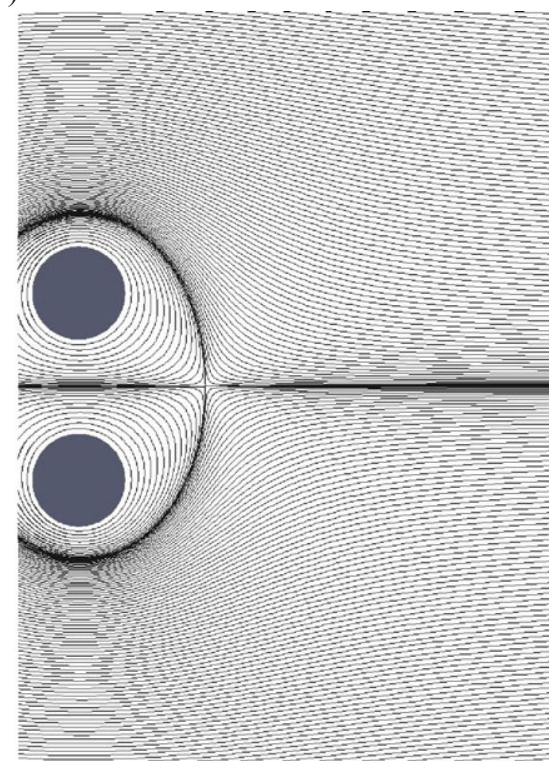

FIGURE 9. (Colour online) Experimental streaklines $(a)$ and computational streamlines (b) showing the existence of the stagnation point and virtual elliptical body for the doublet-like rotational configuration: $g^{*}=1, \operatorname{Re}=150, \Omega=3.1$.

flow is continually accelerated. The force becomes attractive because the fluid velocity between the cylinders is significantly increased due to the induced velocity generated by both the cylinders. The relative increase in the fluid velocity on the outside of the cylinders, the side furthest from the centreline, is noticeably less since it is predominantly induced by the rotation of only a single cylinder. Hence, the $\overline{C_{L}}$ only displays an attractive trend for high rotational rates, where the induced velocity between the cylinder pair becomes sufficiently high to create a pressure imbalance. This effect is amplified at the lowest gap spacing due to the relatively close proximity of the cylinders, and is ultimately responsible for the attractive force observed. It can be hypothesized that an attractive force would be seen for the larger gap spacings at sufficiently high rotational speeds, though further investigation is needed to validate this proposal.

As to the behaviour of the time-averaged drag coefficient, $\overline{C_{D}}$, figure 11 shows that it generally decreases with increasing rotational rate. The results are only shown for one cylinder because the problem is symmetrical in the mean. As found in the case of the mean lift coefficient, the degree of wake interaction and its effect on the mean drag coefficient depends primarily on $g^{*}$ and the effect of Reynolds number is small. For example, in the case of $g^{*}=1$ and $0<\Omega<2.5$, most of the drag reduction comes from the pressure term. For $2.5 \leqslant \Omega<3$, a small net thrust is observed in this 'transitional' range. The net thrust is observed just prior to the creation of the virtual elliptic body. As shown in figure 8, this range corresponds to the coalescence of the front and rear stagnation points of each cylinder into a single stagnation point positioned in a location near that of the rear stagnation point prior to merging. The manipulation of the stagnation points just prior to the creation of the virtual elliptic body is responsible for the pressure imbalance that leads to the generation of a small 

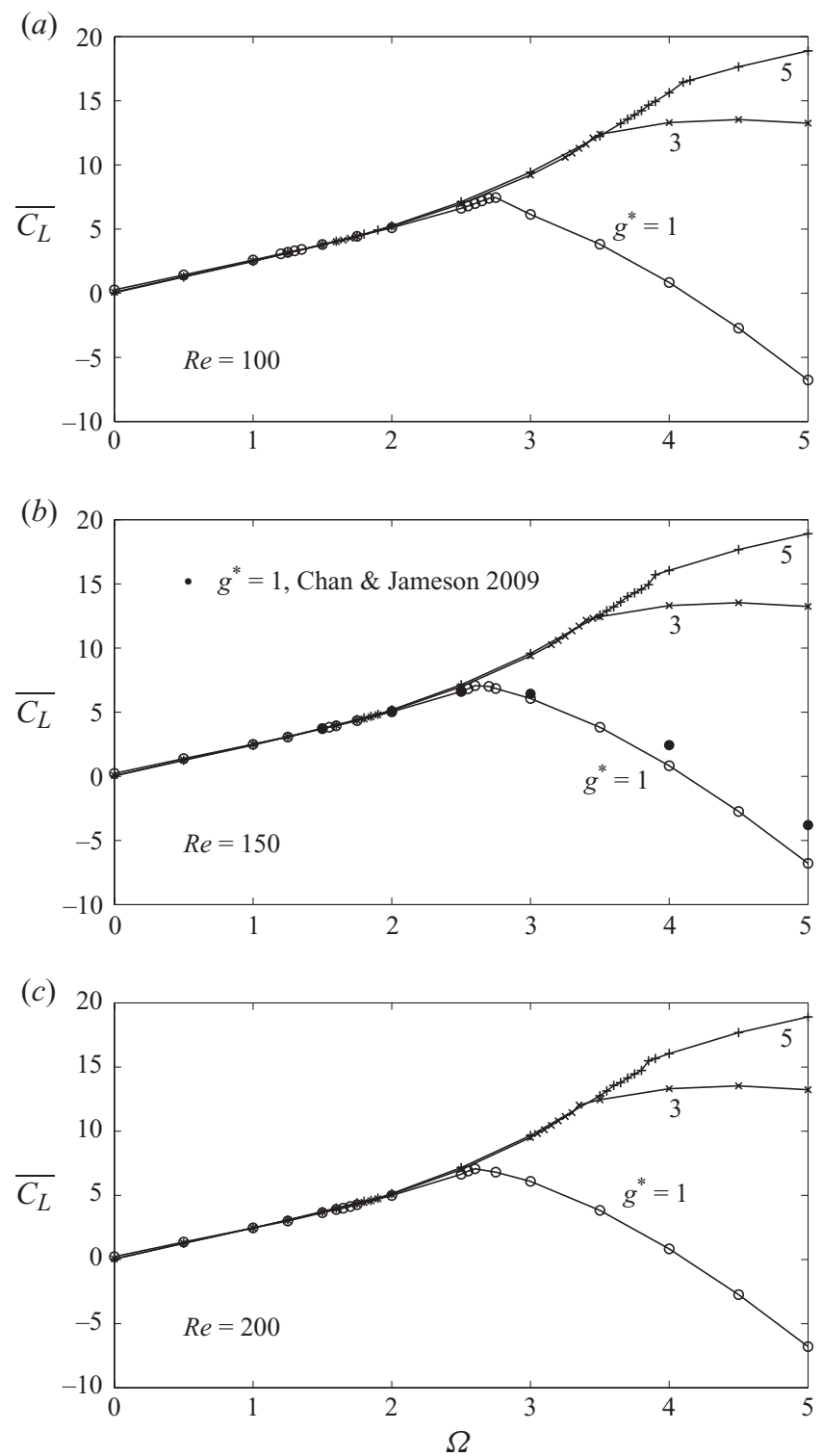

FIGURE 10. $(a)-(c)$ Flow past a doublet-like counter-rotating cylinder: numerically determined time-averaged values of the lift coefficient (upper cylinder).

net thrust. For $3 \leqslant \Omega \leqslant 5$, the pressure drag rises almost linearly with increasing speed while the viscous drag drops linearly at about the same rate, so that the total mean drag is approximately zero. It is in this speed range that the virtual elliptic body forms and grows larger with respect to the increasing speed.

Also plotted on figures 10 and 11 are the results obtained by Chan \& Jameson (2010) for the lift and drag coefficients at $R e=150$ and $g^{*}=1$ using a second-order accurate commercial incompressible flow code. The results are generally in good agreement with the current study, and both investigations show the same trends in the lift and drag behaviour. The current work finds the cross-over speed, where $\overline{C_{L}}$ of 

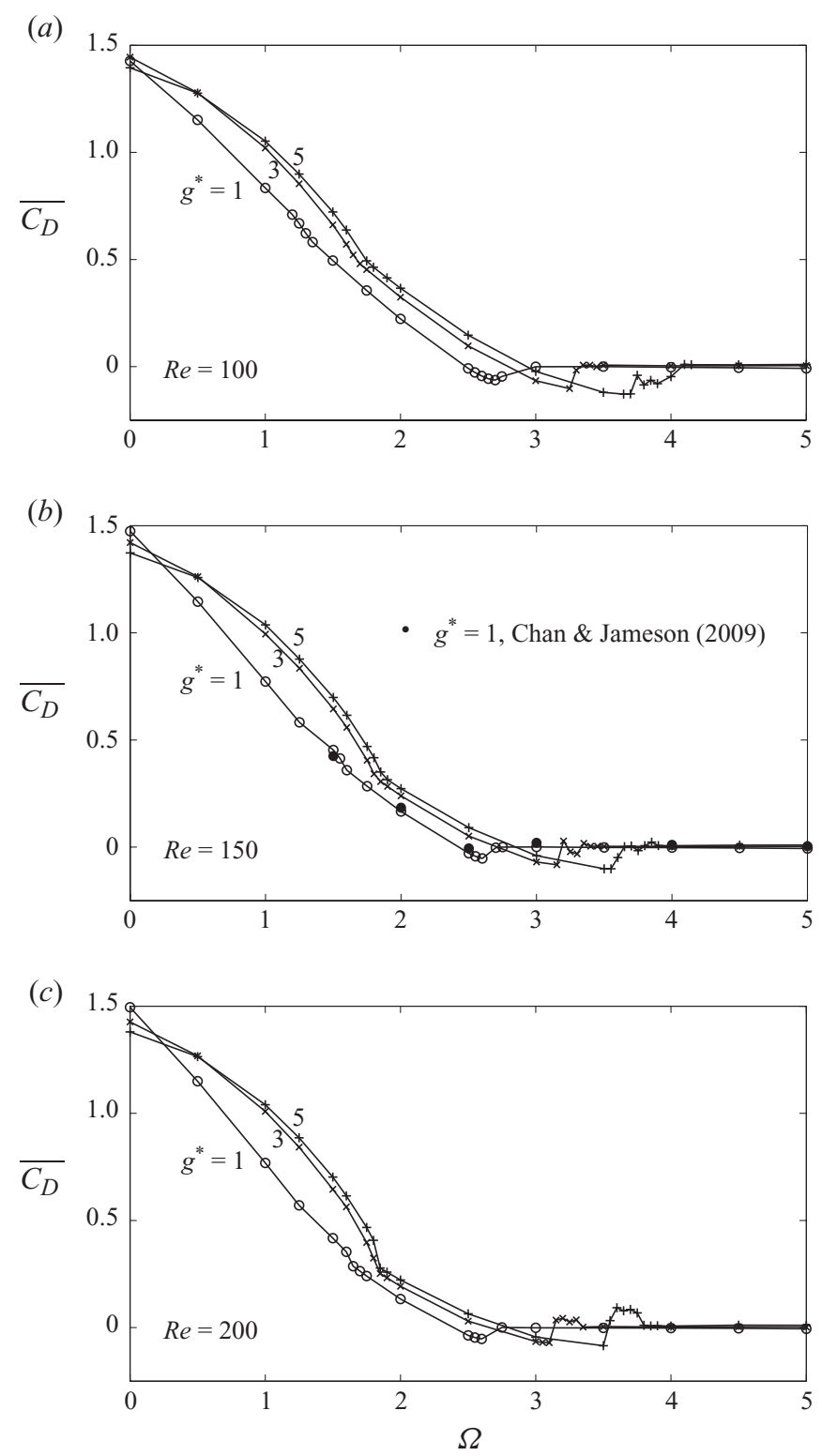

FIGURE 11. (a)-(c) Flow past a doublet-like counter-rotating cylinder: numerically determined time-averaged values of the drag coefficient (upper cylinder).

each cylinder is zero, at $\Omega \approx 4.1$, versus 4.3 from the previous study. This cross-over speed is where the lift force on each cylinder transitions from repelling to attracting. Both studies also find similar characteristics in the drag behaviour in that there is a slight positive net thrust prior to the formation of the closed vortex system, i.e. the virtual elliptic body. In the speed range where the elliptic body is formed, the form drag balances out the viscous drag yielding approximately zero net drag, and the flow pattern resembles a potential doublet. Additionally, the unsteady vortex suppression speed is $\Omega=1.6$ compared to the value of 1.5 found by Chan \& Jameson (2010). Nonetheless, small discrepancies are observed in the lift coefficient where the 
virtual elliptic body is observed $(\Omega \geqslant 3)$. Two primary contributing factors to the differences are the order of accuracy of the method and the effect of compressibility. The present results have been computed with the fourth-order accurate version of the SD scheme at Mach 0.1. Based on the results reported by Ou et al. (2009), we believe the compressibility effect is very small, while fourth-order accuracy is sufficient to produce negligible numerical errors with the mesh that has been used in this work. Accordingly, the current results are believed to be more reliable than those presented by Chan \& Jameson (2010).

For the low-speed range where the pressure term accounts for most of the drag reduction, the mechanism for the mean drag reduction is similar for all gap spacings. The transitional range where a small net thrust is observed occurs at a faster speed with increasing gap size, i.e. at $R e=150,2.5<\Omega<3.2$ for $g^{*}=3$ and $2.5<\Omega<3.6$ for $g^{*}=5$. In general, the larger the gap size, the more the vortex wakes become like those shed from a single spinning cylinder. One very interesting result reported by Mittal \& Kumar (2003) for a single spinning cylinder is the appearance of a second instability in the form of a one-sided vortex shedding mode. In the present case of a pair of counter-rotating cylinders in the doublet configuration, a similar second instability is observed, due to the release of the one-sided vortex that is built up by slow moving vorticity near the stagnation point for each cylinder. This phenomenon is only observed for $g^{*}=3,5$ just prior to the formation of the virtual elliptic body, and is not seen for the smallest gap space, $g^{*}=1$. There is a slight jump in the mean drag when the instability occurs above $\Omega=3$ for $g^{*}=3$ and above $\Omega=3.5$ for $g^{*}=5$. The second instability mode will be discussed in more detail in $\S 4.4$. At higher rotation rates, the virtual elliptic body appears and there is a near-perfect balance between the pressure and viscous drags that produces approximately zero mean drag.

To get a sense of the momentum balance for the experimental and computational work, the streamwise velocity contours and the corresponding velocity profiles are shown in figures 12 and 13 for the case of $g^{*}=1$ and $R e=100$, where the single von Kármán vortex street is present. The velocity profiles are taken at a distance of 10 diameters downstream of the cylinder pair. At lower rotational speeds, a velocity deficit is observed that extends far downstream of the cylinder pair. As the rotational speed increases, the velocity deficit region moves closer to the cylinder pair, until at the highest rotational speeds investigated the deficit region is almost entirely contained in the region where the virtual elliptic body can be found. This results in a very small velocity deficit, indicating that the drag due to momentum flux is negligible in this case. As observed from the experiments, the velocity deficit decreases to almost zero over the range of $\Omega=2-4$, which is consistent with the computational findings.

Experimental drag coefficients could be estimated by integrating the time-averaged velocity profiles at a fixed downstream distance. For the profiles shown in figure 13, the experimentally and computationally determined momentum deficits differed by $4.26 \%, 3.31 \%$ and $2.01 \%$ for $\Omega=1.5, \Omega=2$ and $\Omega=4$, respectively, highlighting the excellent quantitative agreement between the experimental and computational findings.

The Strouhal numbers, are shown in figure 14. Only cases resulting in an anti-phase shedding are shown since this mode produces a distinctly dominant shedding frequency $f$. There is excellent agreement between the results from the $\mathrm{SD}$ computation and those from the experiment. The Reynolds number dependence is most noticeable in going from $R e=100$ to 150 , and less so in going from 150 to 200. The effect of gap spacing is not significant, except in the region of the second 
(a)
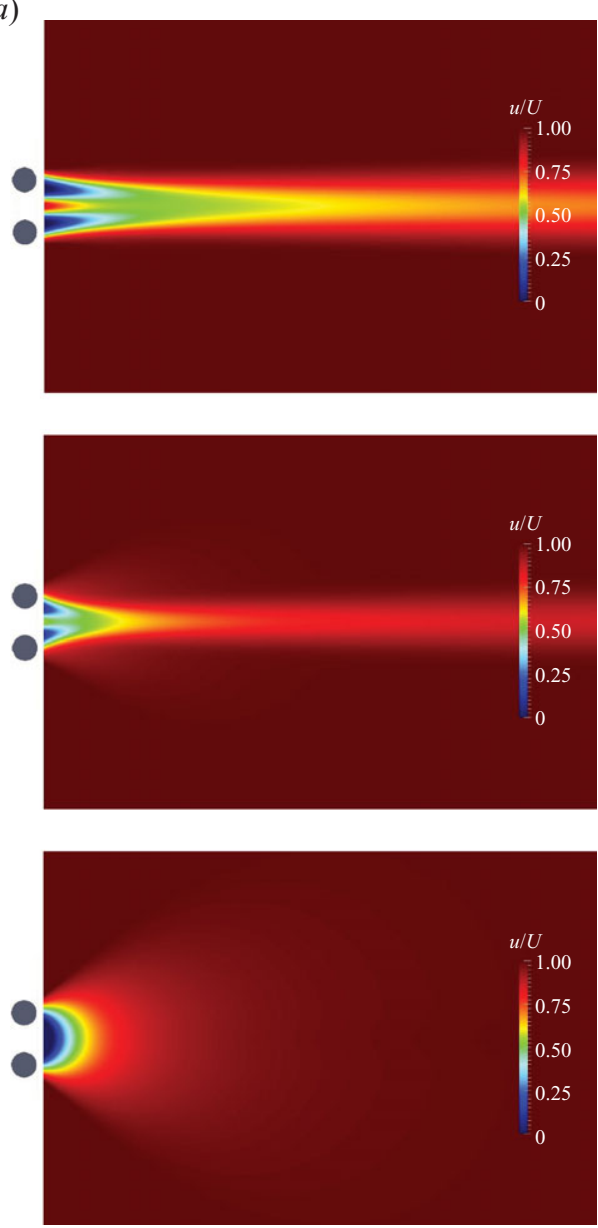

(b)
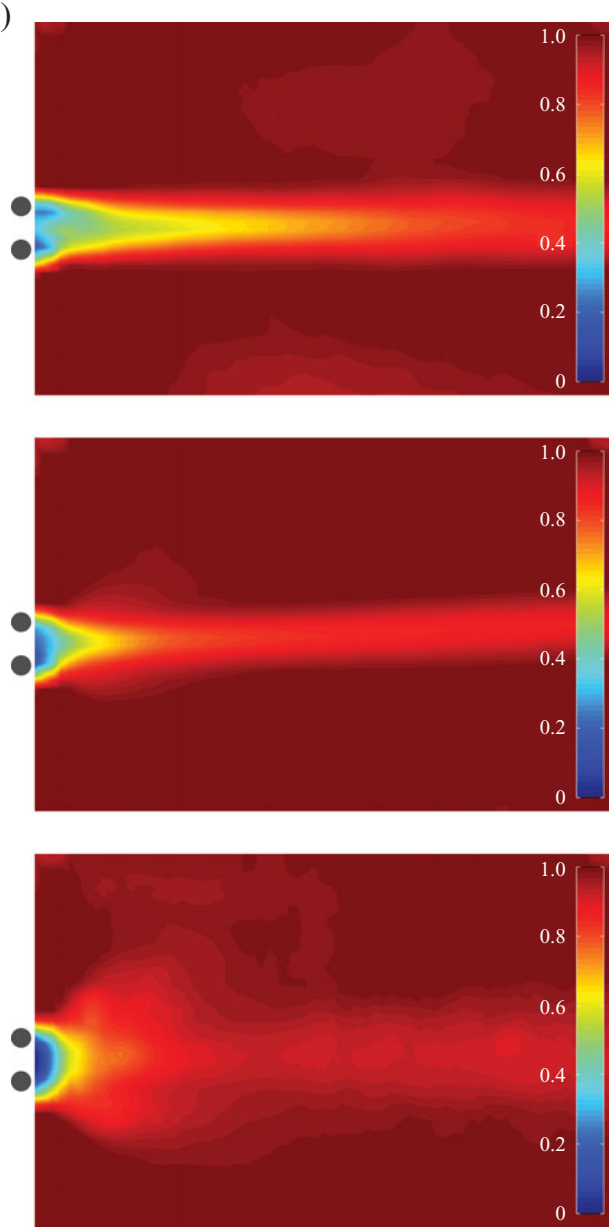

FIGURE 12. Doublet-like rotational configuration: normalized streamwise velocity contours at various rotational speeds for $g^{*}=1, R e=100$. (a) Computational results. (b) Experimental results. First row, $\Omega=1.5$; second row, $\Omega=2$; third row, $\Omega=4$.
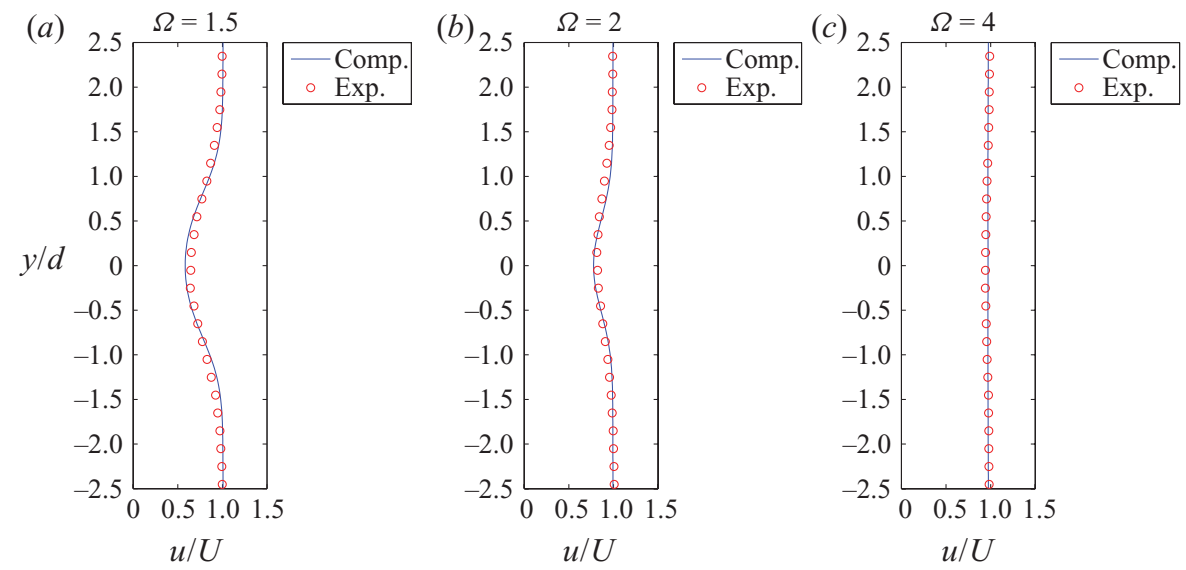

FIgURE 13. (Colour online) (a)-(c) Velocity profiles at $10 d$ downstream from cylinder centre. 

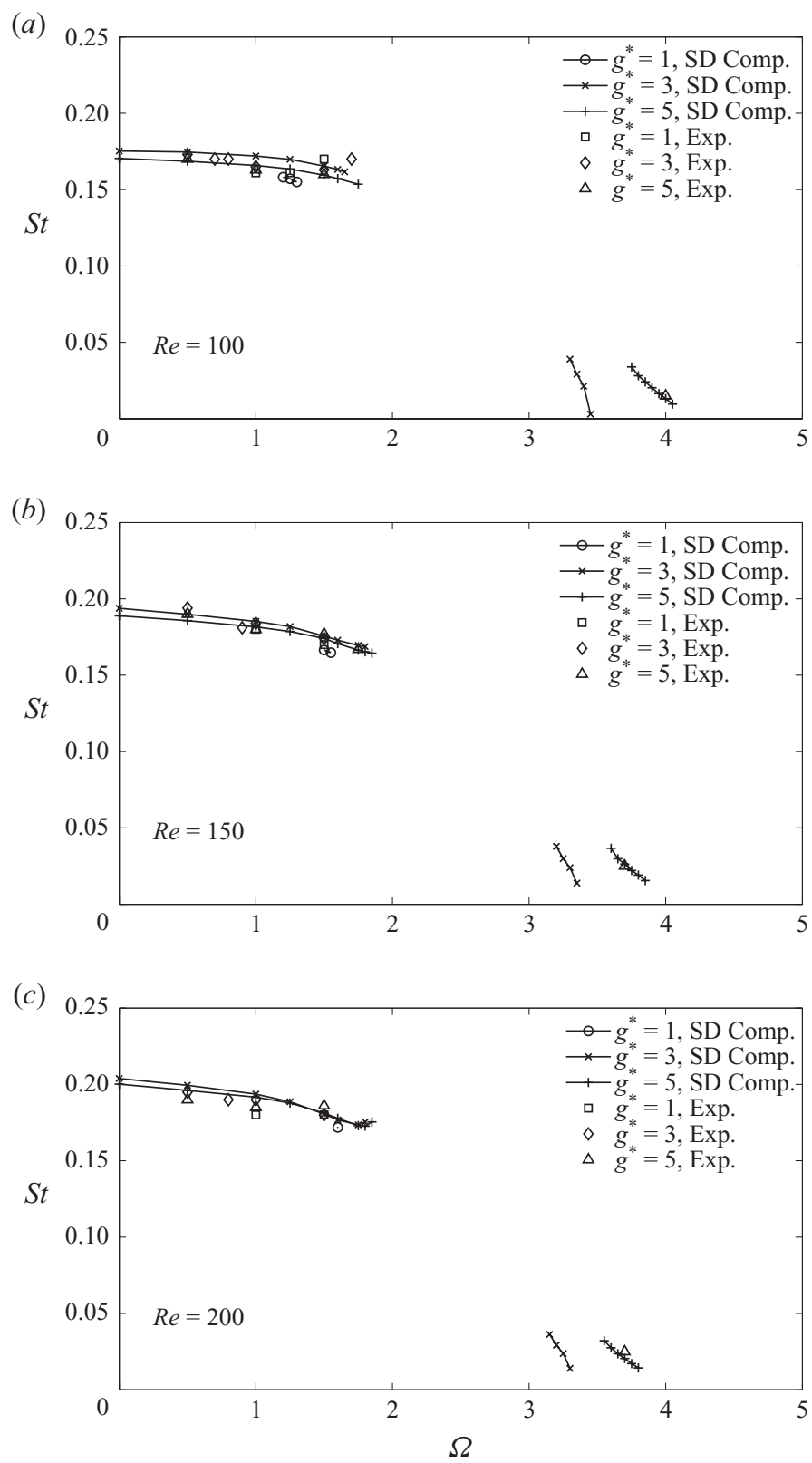

FiguRE 14. (a)-(c) Flow past a doublet-like counter-rotating cylinder: numerically determined Strouhal number (anti-phase shedding only).

instability, i.e. for $3<\Omega<4$. For $g^{*}=1$, the shedding mode is predominantly in-phase for $0<\Omega<1$ and therefore these data points were not included.

\subsection{Reverse doublet-like counter-rotating cylinder pair}

When the cylinder pair is rotated in a reverse doublet configuration (see figure 2), the interaction of the vortex wakes between the two rotating cylinders is substantially reduced because the rotation serves to push the wakes away from one another. Figure 15 compares the computational and experimental vorticity contours and 
(a)
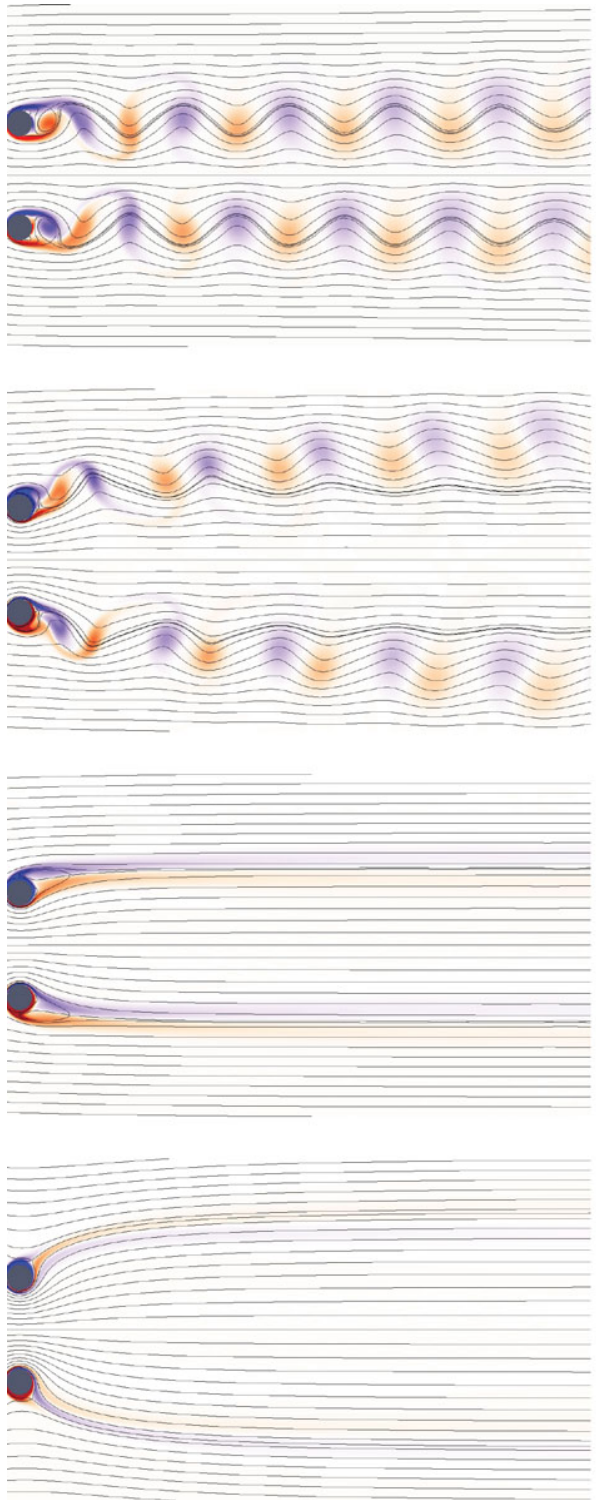

(b)
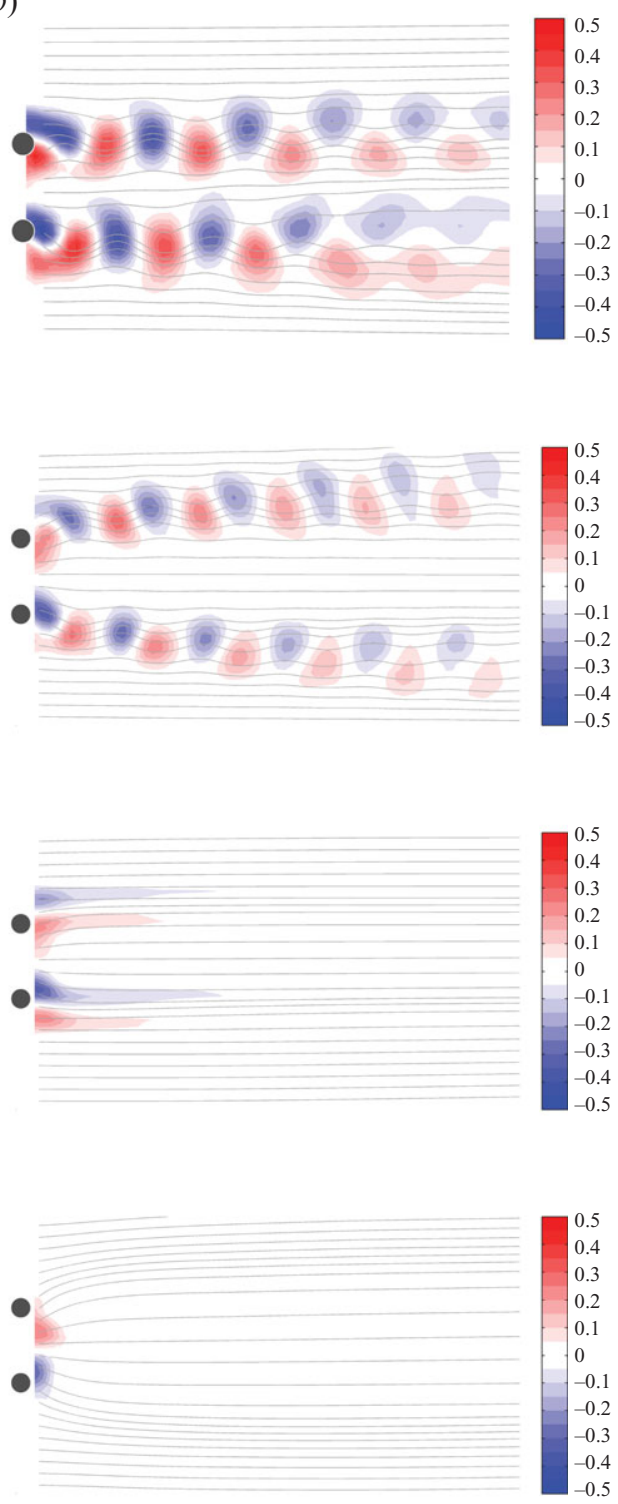

FIGURE 15. Reverse doublet-like rotational configuration: streamlines and vorticity at various rotational speeds for $g^{*}=3, R e=200$. (a) Computational results. (b) Experimental results. First row, $\Omega=0$; second row, $\Omega=2$; third row, $\Omega=2.4$; fourth row, $\Omega=5$. Vorticity contour levels for the computational and experimental results are the same.

streamlines at various rotation rates for $R e=200$ and $g^{*}=3$. It is apparent that the vortex shedding from one cylinder becomes increasingly independent from that of the other cylinder as the rotation rate increases. However, the effect of gap size on the wake interaction is important at the smallest gap $\left(g^{*}=1\right)$ where an in-phase shedding mode exists at all Reynolds numbers for $\Omega<1$. As shown in figure 16, the vortex shedding mode transitions to a stable, anti-phase pattern at $\Omega=1$. This synchronization helps stabilize the vortex street pair and maintain its form further downstream. In this regime, a vortex shed from the upper cylinder is counter-balanced 
(a)

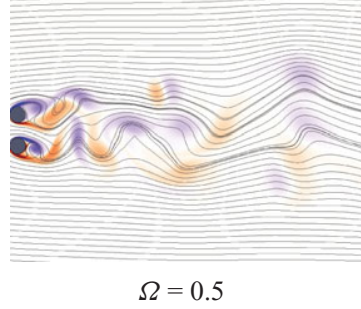

(d)

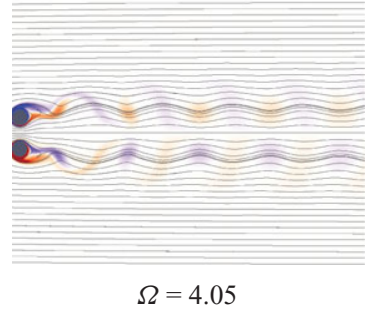

(b)

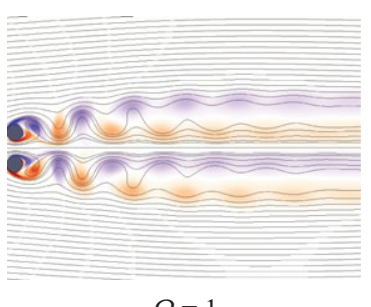

(e)

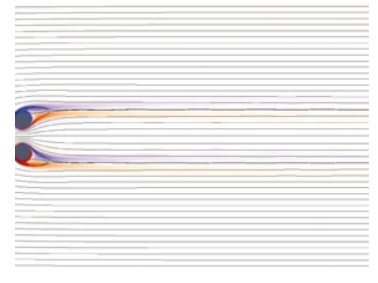

(c)

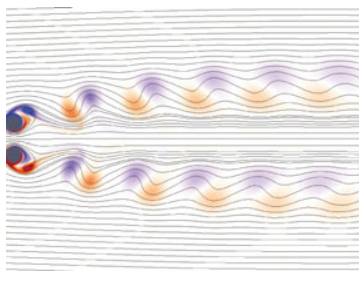

(f)

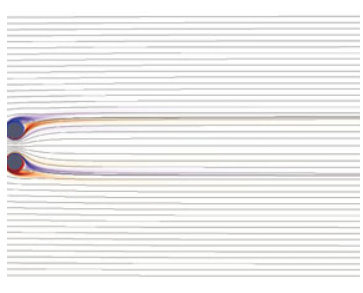

$\Omega=5$

FiguRE 16. $(a)-(f)$ Instantaneous streamlines and vorticity for reverse doublet-like counter-rotating cylinder (computational results): $R e=150, g^{*}=1$. Vorticity contour levels are -0.5 (blue) and 0.5 (red).

(a)

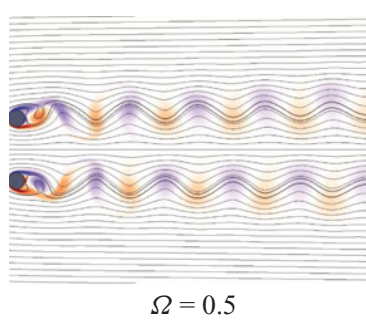

(d)

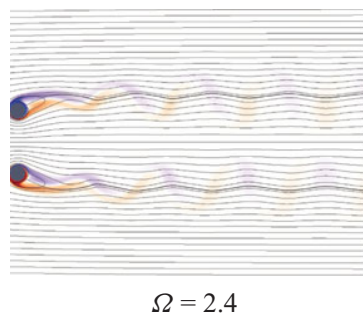

(b)

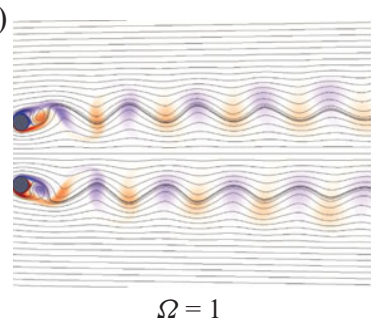

(e)

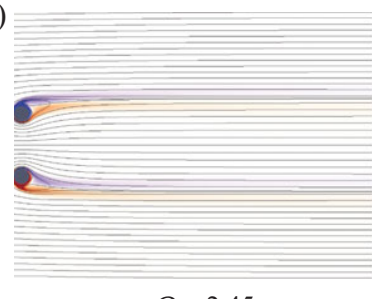

(c)

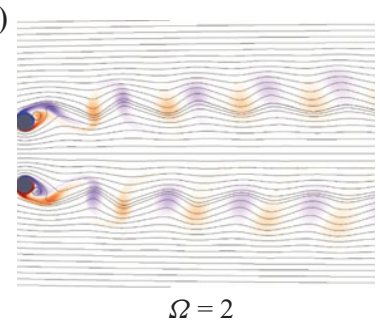

$(f)$

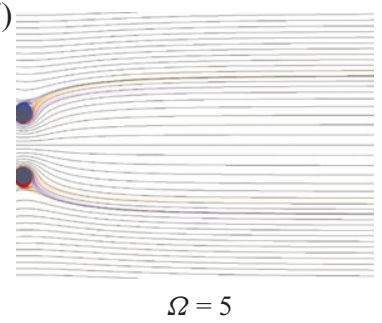

FIGURE 17. $(a)-(f)$ Instantaneous streamlines and vorticity for reverse doublet-like counter-rotating cylinder (computational results): $R e=150, g^{*}=3$. Vorticity contour levels are -0.5 (blue) and 0.5 (red).

by a vortex of opposite sign shed from the lower cylinder. The upper vortex street perfectly mirrors the lower with the line of symmetry being half way between the cylinders, and the strength of each vortex is maintained for long distances downstream. As the gap increases, however, the wake interaction drastically reduces (see figure 17), thus effectively causing the vortex wakes from each cylinder to each behave like that generated by a single rotating cylinder.

The mean lift coefficients, shown in figure 18, are almost independent of gap size and Reynolds number, but the effect of the gap is much more apparent in the drag curves shown in figure 19. It should be noted that as the gap increases, the values of $\overline{C_{D}}$ and 

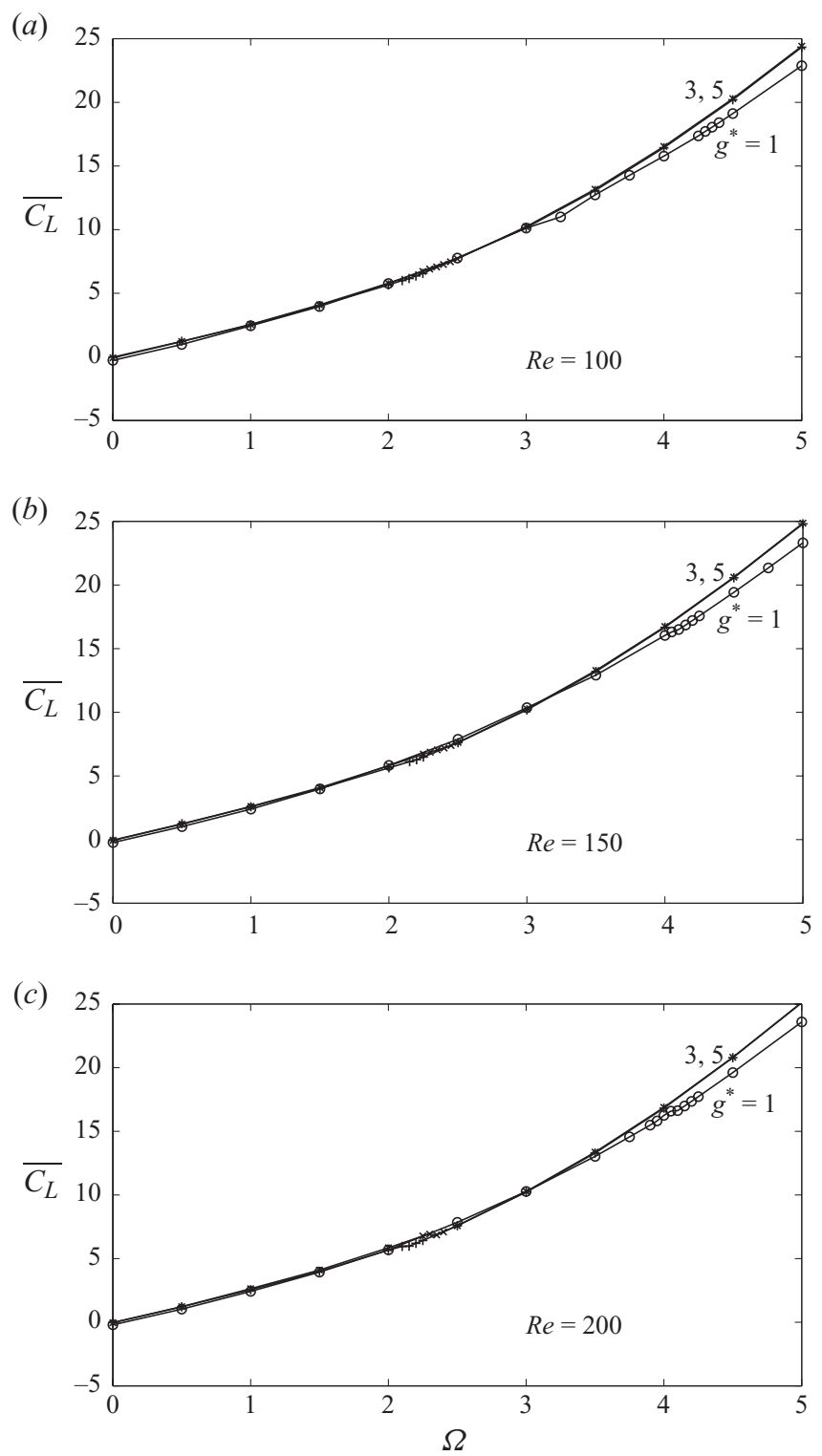

FIGURE 18. (a)-(c) Flow past a reverse doublet-like counter-rotating cylinder: numerically determined time-averaged values of lift coefficient (lower cylinder).

$\overline{C_{L}}$ approach the values of a single rotating cylinder given by Mittal \& Kumar (2003). As observed by Chan \& Jameson (2010), the reverse doublet-like rotation produces an attracting force with a magnitude that increases with the rotation rate. Interestingly, $\overline{C_{D}}$ for $g^{*}=1$ shows a non-monotonic behaviour with the rotational rate. This is the result of a transition from an in-phase shedding mode to an anti-phase shedding mode at $\Omega \approx 1$ for the lowest gap spacing in this rotational configuration. The effective width of the wake increases for the in-phase mode due to its non-sinusoidal nature, causing $\overline{C_{D}}$ to increase until the stable anti-phase mode is achieved. The Strouhal numbers plotted in figure 20 show little dependence on the gap spacing and rotation 

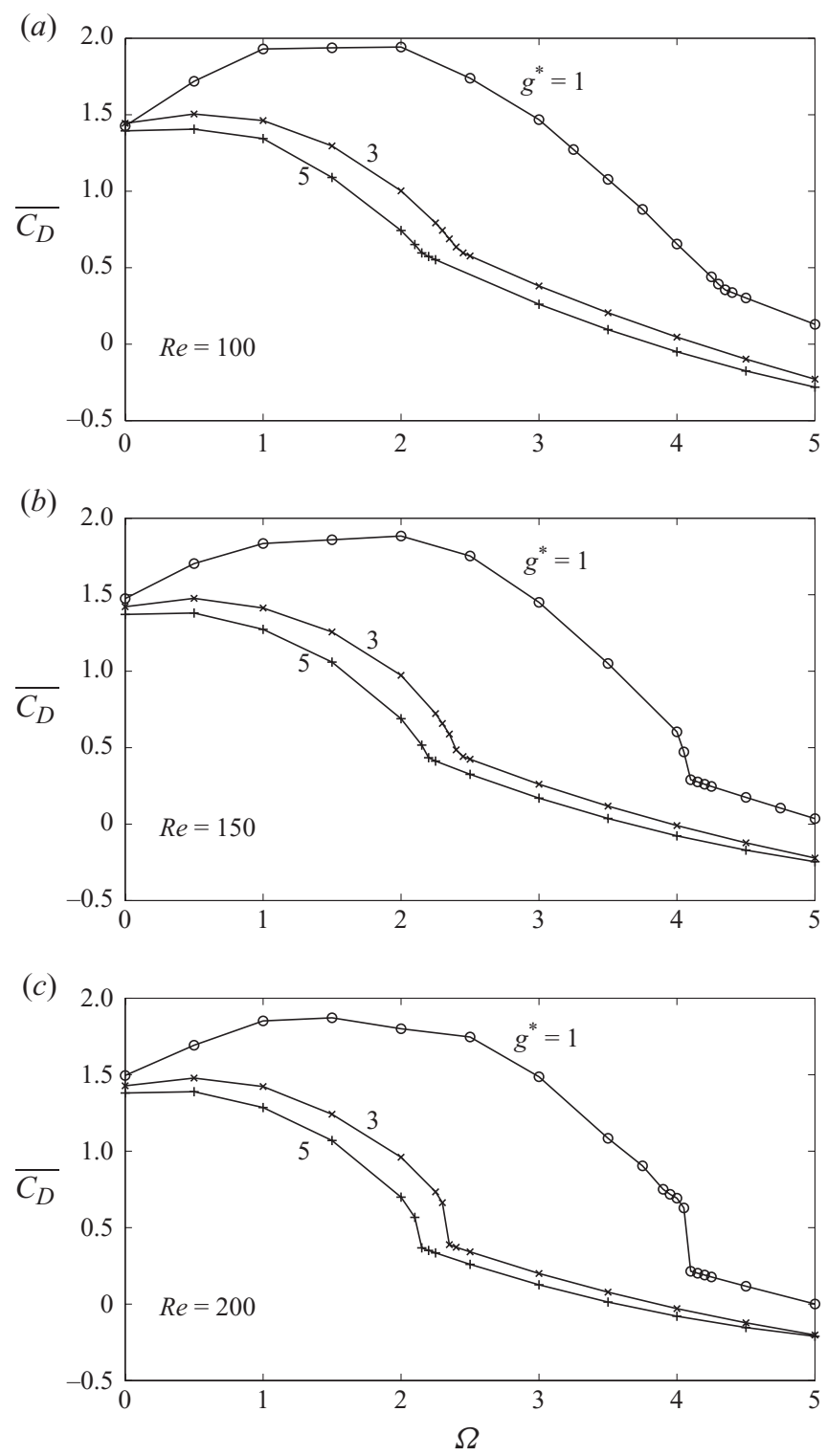

FIGURE 19. (a)-(c) Flow past a reverse doublet-like counter-rotating cylinder: numerically determined time-averaged values of drag coefficient (lower cylinder).

rate, although there is a distinct Reynolds number dependence, especially at the lower rotation rates. Also, the results obtained from the SD computation generally agree very well with the experimental results.

\subsection{Unsteady vortex suppression}

The standard deviation of the lift and drag coefficients for the doublet-like rotation are shown in figure 21; the corresponding values for the reverse doublet-like are shown in figure 22. The standard deviation gives a reasonably good prediction of the critical rotation speed, $\Omega_{\text {crit }}$, where the unsteady wakes are suppressed, in that steady flow is observed when the standard deviation value is zero. Experimentally, $\Omega_{\text {crit }}$ 

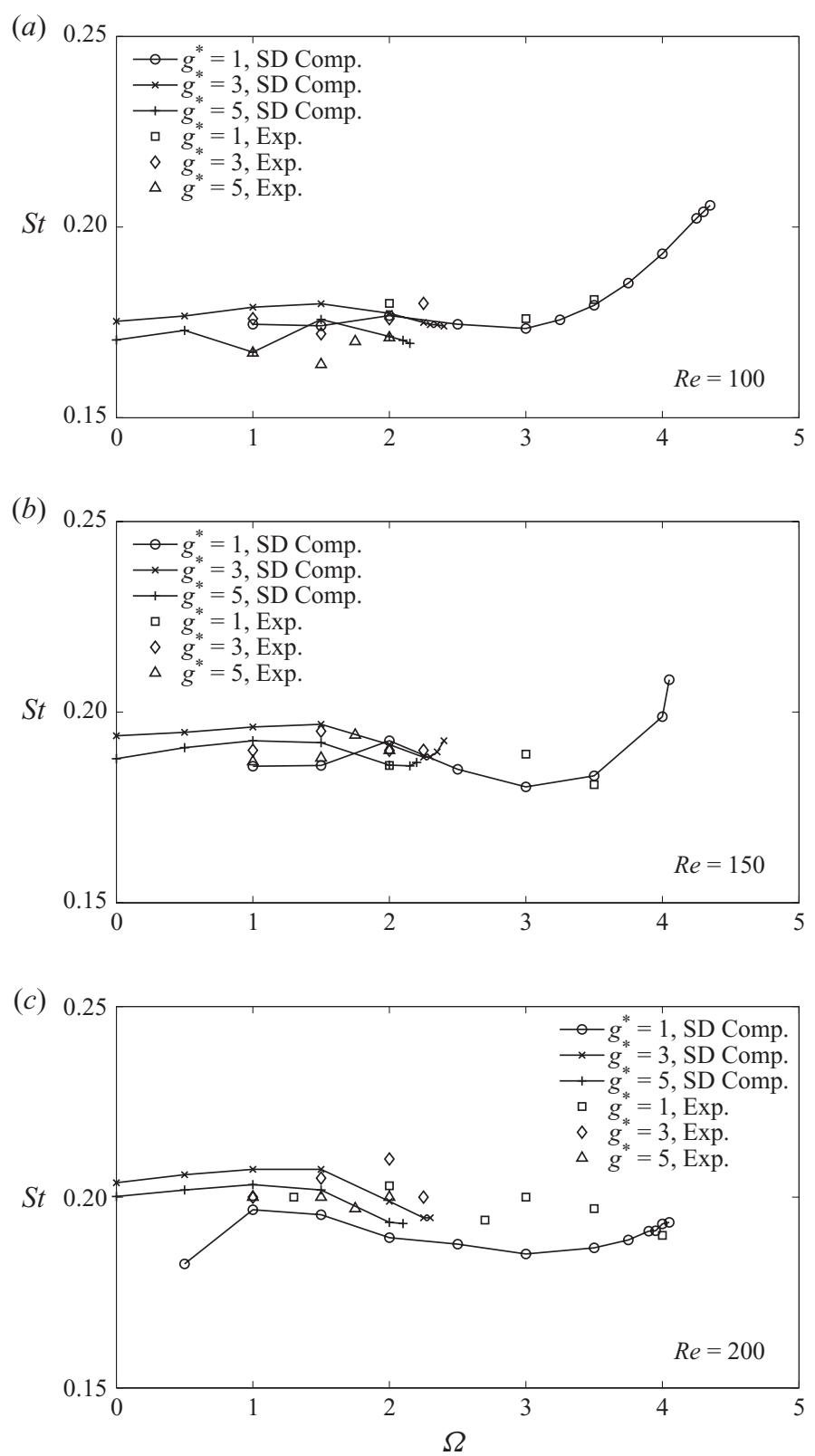

FIGURE 20. (a)-(c) Flow past a reverse doublet-like counter-rotating cylinder: numerically determined Strouhal number (anti-phase shedding only).

was determined when the FFT of the time-resolved velocity field yielded a shedding frequency of less than $5 \%$ of the shedding frequency for cylinders rotating at speeds just below suppression. The estimated uncertainty in the experimentally determined $\Omega_{\text {crit }}$ is at most $9 \%$.

The computationally determined values of $\Omega_{\text {crit }}$ are compared with the experimental values in figure 23, and are summarized in table 1 . There is excellent agreement for all cases that were investigated, except for the reverse doublet configuration at 
$g^{*}=1$ where the computational results are approximately 10-20\% larger than the experimental ones. Observations of the particle motion in the DPIV experiments suggest that at higher rotational speeds the flow exhibits a noticeable degree of three-dimensionality, particularly in the case of $g^{*}=1$. This was also confirmed by noting that the two-dimensional continuity equation deviated slightly from zero in the near wake of the cylinders for low gap spacings and high rotational speeds. Thus, it is possible that this discrepancy occurs because the computations used in this work were strictly two-dimensional. Nevertheless, the critical rotational speed monotonically decreased with increasing gap spacing, and the data suggest that $\Omega_{\text {crit }}$ depends more strongly on gap spacing than on Reynolds number.

For the doublet-like cylinder rotation (figure 23), $\Omega_{\text {crit }}<1.9$ in all cases, and its value monotonically increases with increasing gap spacing and Reynolds number. The results presented by Yoon et al. (2007) and the current investigation only have one point of overlap $\left(R e=100, g^{*}=3\right)$, but in both studies the critical vortex suppression was found to be 1.7 . However, as seen in the figure 21 , there is a small region of instability at speeds above $\Omega_{\text {crit }}$. This second instability mode occurs in the doubletlike configuration at $\Omega_{\text {crit }} \approx 3.25$ for $g^{*}=3$ and $\Omega \approx 3.75$ for $g^{*}=5$, but is suppressed for $g^{*}=1$. As indicated earlier, this instability is related to the same phenomenon observed in the case of a single rotating cylinder, as investigated by Mittal \& Kumar (2003). It will be discussed further in $\S 4.4$.

Rotation of the cylinder pair in the reverse doublet-like configuration (figure 23) reduces the interaction of the vortex streets resulting in higher critical rotation speeds (that is, $\Omega_{\text {crit }}>2$ ). This is in contrast to the doublet-like configuration, increasing the gap spacing reduces the vortex suppression speeds for the reverse doublet-like configuration. For $g^{*}=1$, the critical speed is as high as $\Omega_{\text {crit }}=4$.4, indicating that the proximity of a nearby cylinder promotes the instability of the wake. At the largest spacing, $g^{*}=5, \Omega_{\text {crit }}$ collapses near 2.3 for all Reynolds numbers. Also, an increase in the gap spacing results in a monotonic decrease in the critical speed.

When the results for both rotational configurations are compared (figure 23), we see that they appear to converge to a single value with increasing gap size. As noted earlier, this is not surprising, in that with increasing gap size the interaction between the two cylinder wakes diminishes, and in the limit we would expect the two wakes to become independent and to have $\Omega_{\text {crit }}$ approach the value to suppress vortex formation in the wake of a single rotating cylinder. Kang, Choi \& Lee (1999) found that for a single rotating cylinder at $R e=100, \Omega_{\text {crit }}=1.8$, and at $R e=160$ it was 1.9 , and Mittal \& Kumar (2003) reported the first critical suppression speed for a single cylinder to be 1.91 at $R e=200$. These values are very similar to the convergent value suggested here, which gives $\Omega_{\text {crit }} \approx 1.9$ at $R e=100$, and approximately 2 at $R e=150$.

\subsection{The second region of instability}

In figure 21, we saw evidence for a small region of instability at speeds above $\Omega_{\text {crit }}$ for the doublet-like configuration that depends on the gap spacing. Mittal \& Kumar (2003) observed a similar second instability in the case of a single rotating cylinder, and they postulated that it occurs because the balance between the advection and diffusion of vorticity is disturbed. With rotation in a counter-clockwise direction in the regime of the second instability speed (i.e. $4.35<\Omega<4.75$ for $R e=200$ ), they observed the build up of positive vorticity in the slow moving flow near the stagnation point in the near wake that, once it gathered enough strength, shed at a lower frequency than that seen in the regime of the first instability. 
(a)
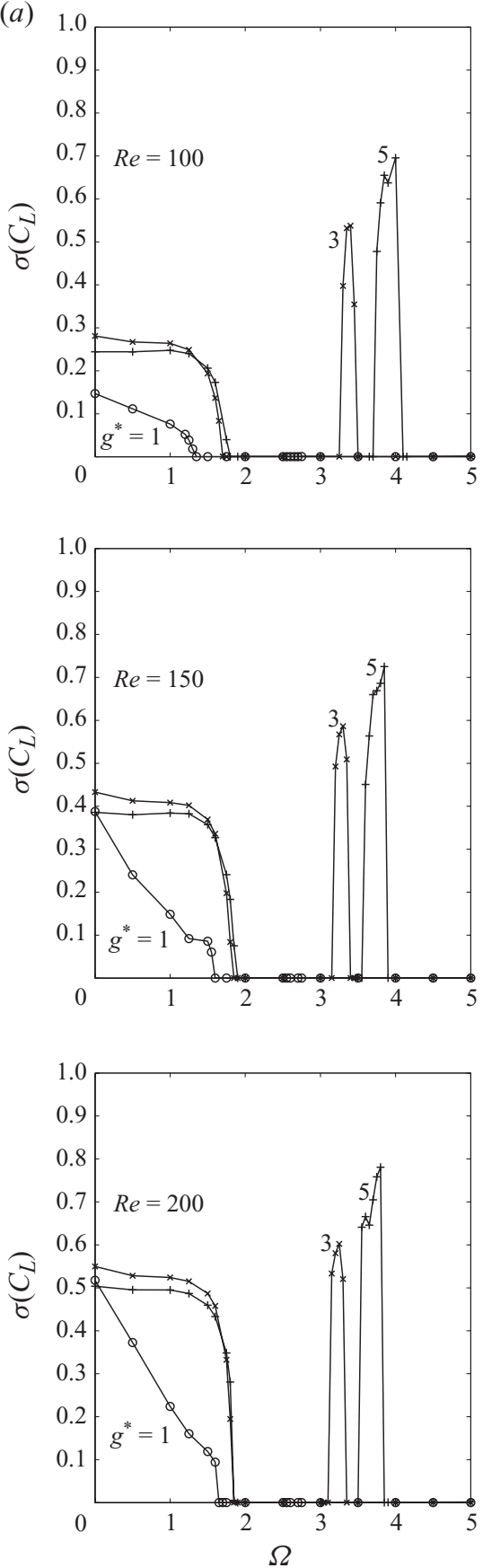

(b)
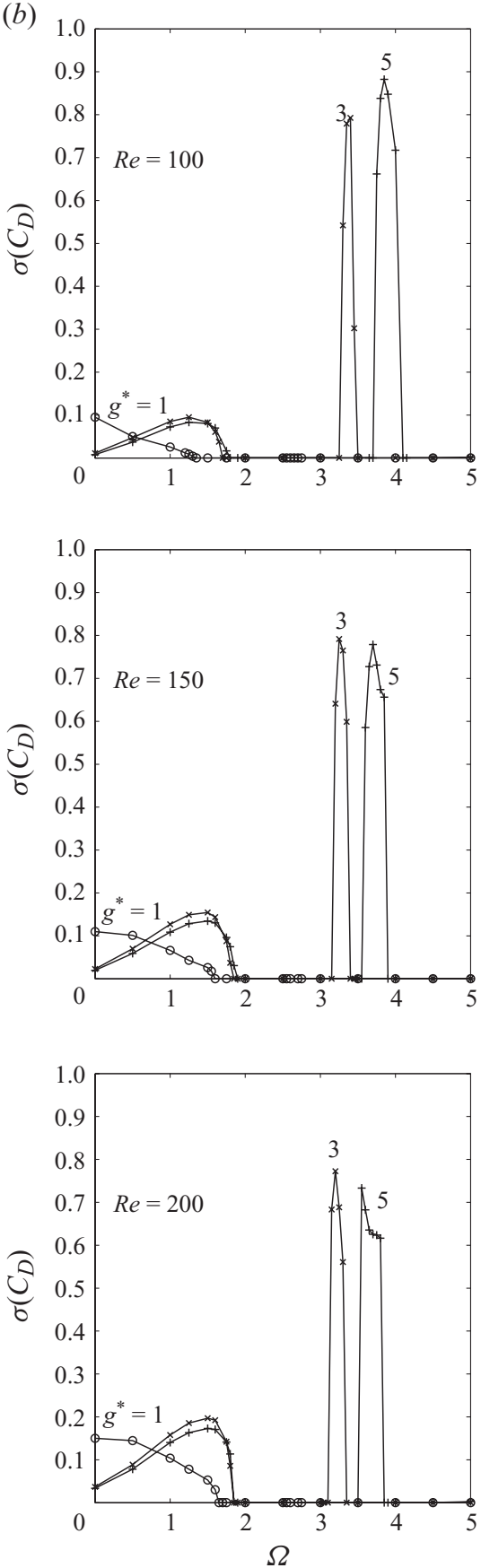

FIGURE 21. Flow past a doublet-like counter-rotating configuration: standard deviation of the coefficients of lift $(a)$ and drag $(b)$, obtained numerically.

In the current work, the computations and experiments indicate that when the gap is small (i.e. $g^{*}=1$ ), the interaction between the two vortex streets prevents the second instability from occurring. When the vortex shedding is suppressed at sufficiently high spin rates, there are two pairs of stationary stagnation points associated with the 

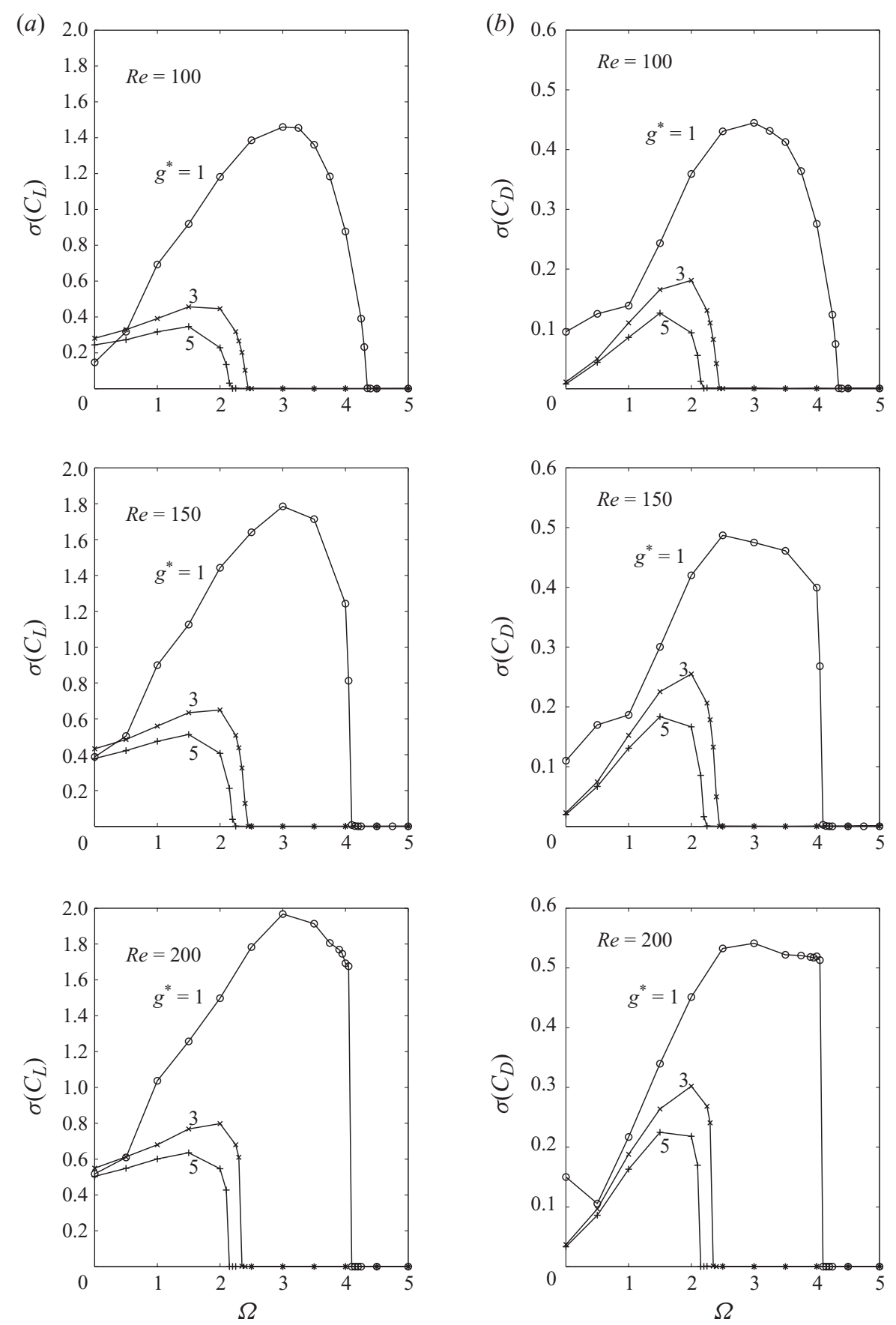

FIGURE 22. Flow past a reverse doublet-like counter-rotating configuration: the standard deviation of coefficients of lift $(a)$ and drag $(b)$.

positive (red) and negative (blue) vortices from each cylinder, as shown on the left column of figure 8. Each front stagnation point (that is, upstream of the cylinder centres) moves outward radially with increasing spin rate (that is, with increasing circulation) and its location follows the closed streamline around the cylinder body. 


\begin{tabular}{cccc}
\multicolumn{4}{c}{ Doublet-like configuration } \\
$R e$ & $g^{*}=1$ & $g^{*}=3$ & $g^{*}=5$ \\
100 & $1.35(1.32)$ & $1.70(1.72)$ & $1.80(1.79)$ \\
150 & $1.60(1.61)$ & $1.85(1.86)$ & $1.90(1.94)$ \\
200 & $1.65(1.70)$ & $1.85(1.88)$ & $1.90(1.94)$ \\
\multicolumn{4}{c}{} \\
100 & Reverse doublet-like configuration \\
150 & $4.40(3.84)$ & $2.45(2.52)$ & $2.20(2.24)$ \\
200 & $4.10(3.71)$ & $2.45(2.53)$ & $2.25(2.28)$ \\
& $4.10(3.53)$ & $2.40(2.46)$ & $2.15(2.27)$
\end{tabular}

TABLE 1. Critical rotational rate to suppress vortex formation, experimental values in parentheses.

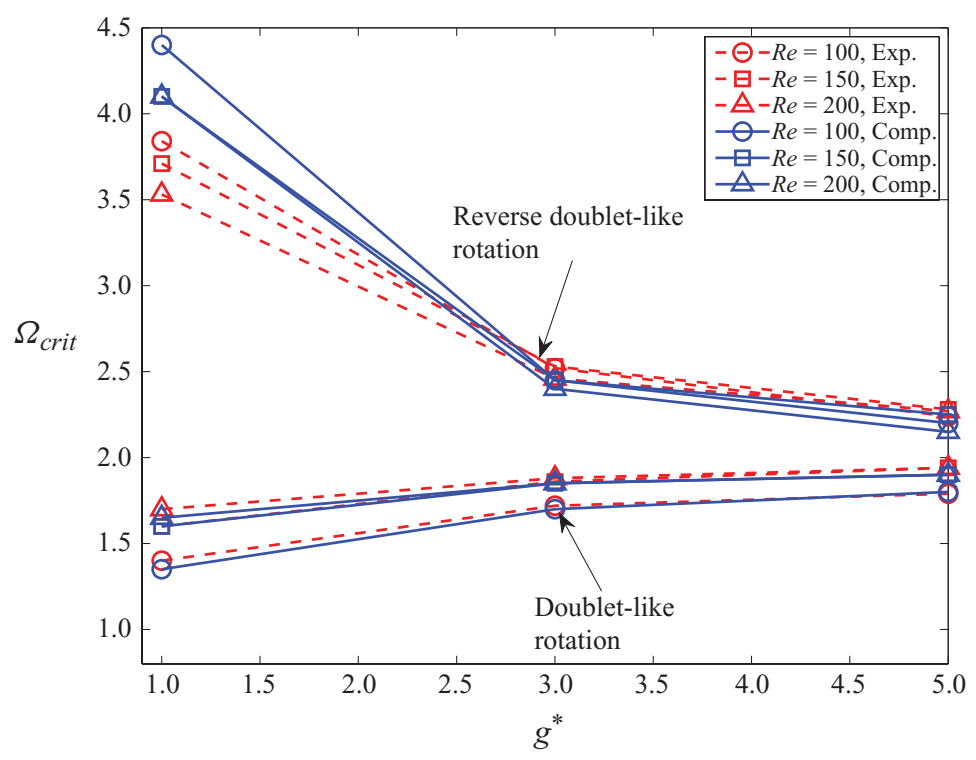

FIGURE 23. (Colour online) Critical rotation speed for vortex suppression.

In contrast, each rear stagnation point (i.e. downstream of the cylinder centres) moves inwards towards the front stagnation point as the spin rate increases. At some point, the two closed vortex systems merge into one, and the two stagnation points combine into a single point at a location near that of the rear stagnation point prior to merging (centre column of figure 8). By increasing the spin rate further, each closed vortex system is stretched to resemble a tear drop shape, and the stagnation point progresses towards the horizontal centreline. It is at this point where we observe that the second instability occurs prior to the appearance of the virtual elliptic body.

The relatively large gap sizes for which this instability occurs, i.e. $g^{*}=3$ and 5, effectively cause the vortex street from each cylinder to appear similar to that produced by a single rotating cylinder. However, the interaction between the two vortex streets is apparently sufficient to lower the spin rate at which the instability appears when compared to the case of a single rotating cylinder. For the doublet-like rotation, we observe that for $\operatorname{Re}=200, g^{*}=5$, the second instability occurs at $3.55 \leqslant \Omega \leqslant 3.8$ over 
(a)

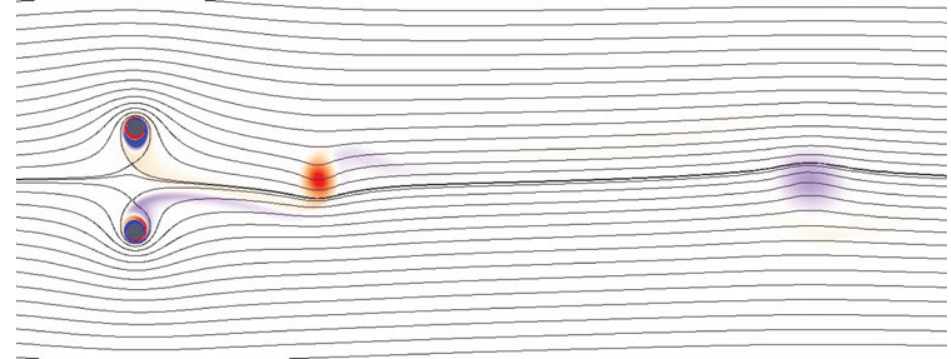

(b)

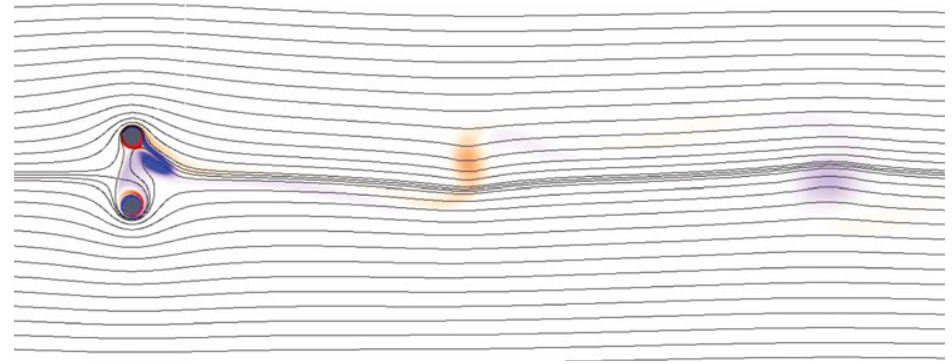

FIGURE 24. Instantaneous streamlines and vorticity for the second instability of the doublet-like counter-rotating configuration (computational results): (a) $R e=200, g^{*}=5, \Omega=3.75$; (b) $R e=200, g^{*}=3, \Omega=3.25$. Vorticity contour levels are -0.1 (blue) and 0.1 (red).

a Strouhal number ranging from 0.0143 to 0.0321 . For $R e=200, g^{*}=3$, it occurs at $3.65 \leqslant \Omega \leqslant 3.85$ with $0.0155 \leqslant S t \leqslant 0.0298$. As was shown in figure 14 , there are some slight differences in the values of $S t$ and $\Omega$ corresponding to the second instability at different Reynolds numbers and gap spacings. For example, consider the vorticity and streamlines of the second instability shedding mode for $g^{*}=3$ and $g^{*}=5$ shown in figure 24. The red vortex was shed from the upper cylinder while the blue one was shed from the lower cylinder. The decreasing distance between successive vortex cores with increasing Strouhal number reflects the higher shedding frequency. Also note the excellent agreement between the computation and experiment shown in figure 25 for $R e=100, g^{*}=5$ and $\Omega=4$.

In the reverse doublet-like case, the second instability does not occur in the speed range of our investigation because in the first steady flow region the positive and negative vortices in the vicinity of the cylinder bodies are less likely to affect one another. The front and rear stagnation points are produced away from the centreline and in opposing pairs. Consequently, it appears that this configuration helps delay the merging of the front and rear stagnation points. Figure 26 illustrates the progression from unsteady (left column) to steady flow (centre and right columns) for $R e=150$ for different gap sizes. We see that at $\Omega=5$ for $g^{*}=3$ and 5 , only a single stagnation point is formed for each cylinder. A qualitative comparison between figure 26 in the present work and figure 8 from Mittal \& Kumar (2003) suggests that the delay in the merging of the front and rear stagnation points caused by the presence of a second counter-rotating cylinder retards the onset of the region of secondary instability. It remains an open question as to whether the secondary instability exists at higher rotational speeds than those examined here. 
(a)

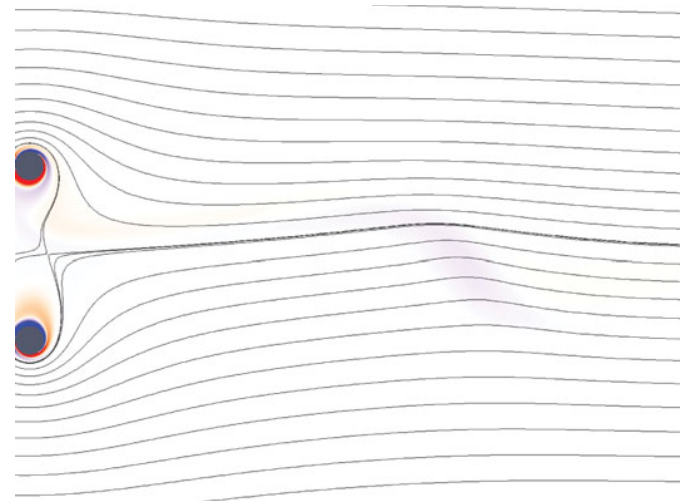

(b)

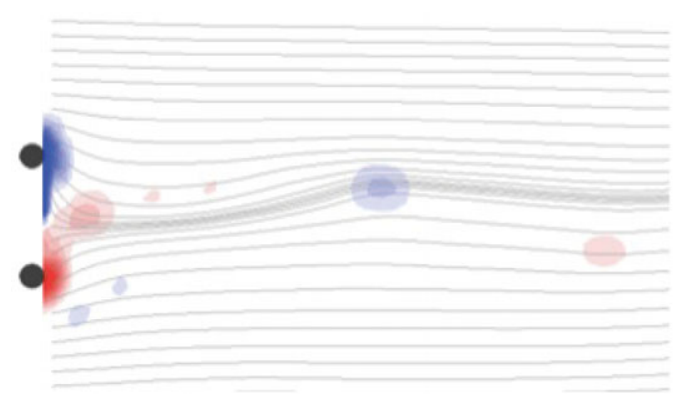

FIGURE 25. Instantaneous streamlines and vorticity for the second instability of the doublet-like counter-rotating configuration, $R e=100, g^{*}=5, \Omega=4$ : $(a)$ computational; (b) experimental. Vorticity contour levels are -0.5 (blue) and 0.5 (red).

\subsection{Power consumption for rotating the cylinder pair}

The possible benefits of unsteady wake suppression bear a cost in terms of the power consumption required to overcome the translational aerodynamic drag as well as to maintain the rotational motion of the cylinder pair. This can be analysed in terms of the time-averaged power coefficient defined by

$$
\overline{C P}=\overline{C_{D}}+\frac{\left|\overline{C_{M}}\right| \omega d}{2 U},
$$

where $\overline{C_{M}}$ is the time-averaged moment coefficient calculated from the moment about each cylinder centre. Figure 27 shows $\overline{C P}$ (of the upper cylinder) plotted against rotational speed for both the doublet-like and reverse doublet-like configurations in comparison to the single rotating cylinder at $R e=200$. The trends are similar for $R e=100$ and 150. It is observed that in both rotational configurations, as the gap size increases, and not surprisingly, the curves converge towards that of the single rotating cylinder. As the gap size decreases, it is advantageous, or more efficient, to rotate the cylinder pair in the doublet-like configuration due to a decrease in both the aerodynamic drag contribution, $(\overline{C P})_{D}$, and the required torque, $(\overline{C P})_{M}$, as shown in figure 28 . Note that the aerodynamic drag contribution to $\overline{C P}$, shown in figure $28(a)$, is essentially a replot of $\overline{C_{D}}$ for $R e=200$ (figures 11 and 19).

An interesting phenomenon is observed in the doublet-like case where there appears to be a noticeable change in the slope of the power coefficient in the transitional range prior to the formation of the elliptical body. This is particularly so for $g^{*}=1$, where 
(a)

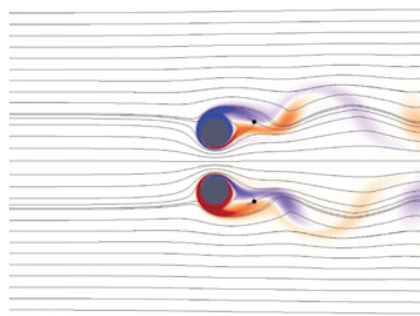

$\Omega=4.05$

(b)

(c)

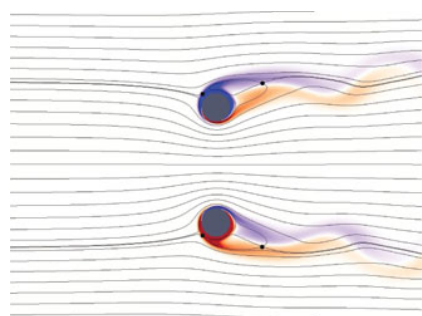

$\Omega=2.4$

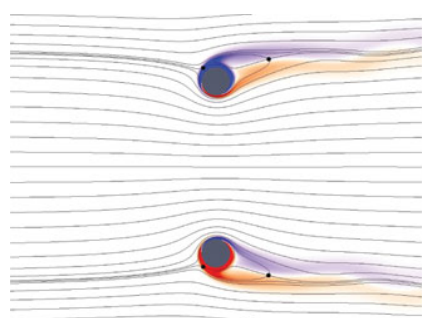

$\Omega=2.2$

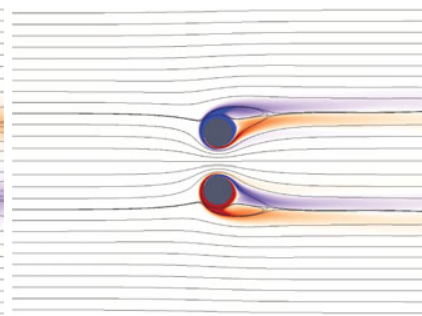

$\Omega=4.1$

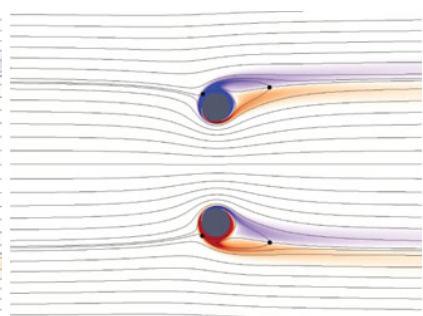

$\Omega=2.45$

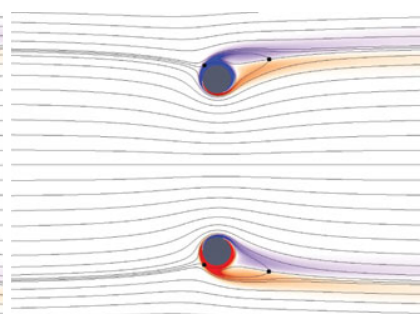

$\Omega=2.25$

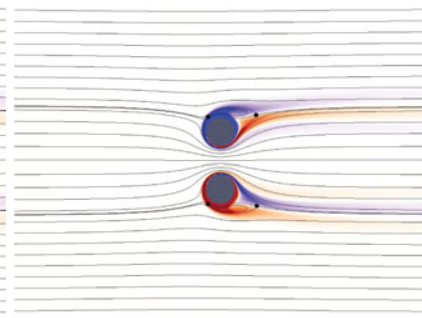

$\Omega=5$

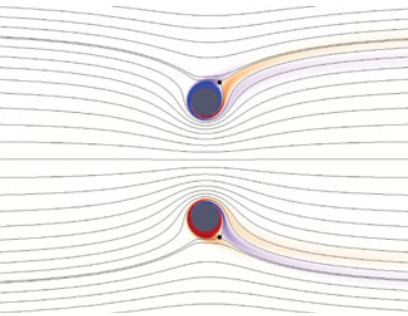

$\Omega=5$

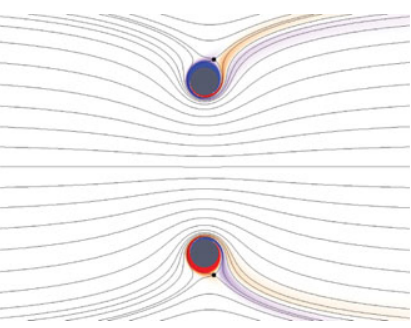

$\Omega=5$

FIGURE 26. Flow past a reverse doublet-like counter-rotating configuration: instantaneous streamlines and vorticity at various rotational speeds (computational results). Stagnation points are represented by ' $\bullet$ '. (a) $\operatorname{Re}=150, g^{*}=1$; (b) $\operatorname{Re}=150, g^{*}=3$; (c) $\operatorname{Re}=150, g^{*}=5$. Vorticity contour levels are -0.5 (blue) and 0.5 (red).

a clear discontinuity in the spinning torque curve is observed in figure $28(b)$, such that there is a sudden jump in $(\overline{C P})_{M}$ at $\Omega=2.6$. In this rotational regime, the stagnation points transition from being vertically to horizontally aligned (i.e. from the middle column to right column of figure 8) and the flow between the cylinder pair reverses direction, opposing the oncoming free stream velocity. It may be that in this transitional regime, as the flow between the cylinder pair becomes stagnant before reversal, there is a significant reduction in the torque required to rotate the cylinders. Once the flow has reversed direction and the virtual elliptic body has formed, the cylinders must continually accelerate the fluid particles contained between the cylinder pair, thus returning the power coefficient to a monotonically increasing trend. This behaviour occurs over a very short interval in $\Omega$, and a further investigation of this flow mechanism is required to better understand the nature of this regime. It may also be possible that three-dimensionality plays an ever increasing role at lower gap spacings, potentially highlighted by the fact that such a large discontinuity in power coefficients is only seen for $g^{*}=1$. For $g^{*}=3$ and 5 , the range of speeds where the slope changes also coincides with the occurrence of the second instability. 


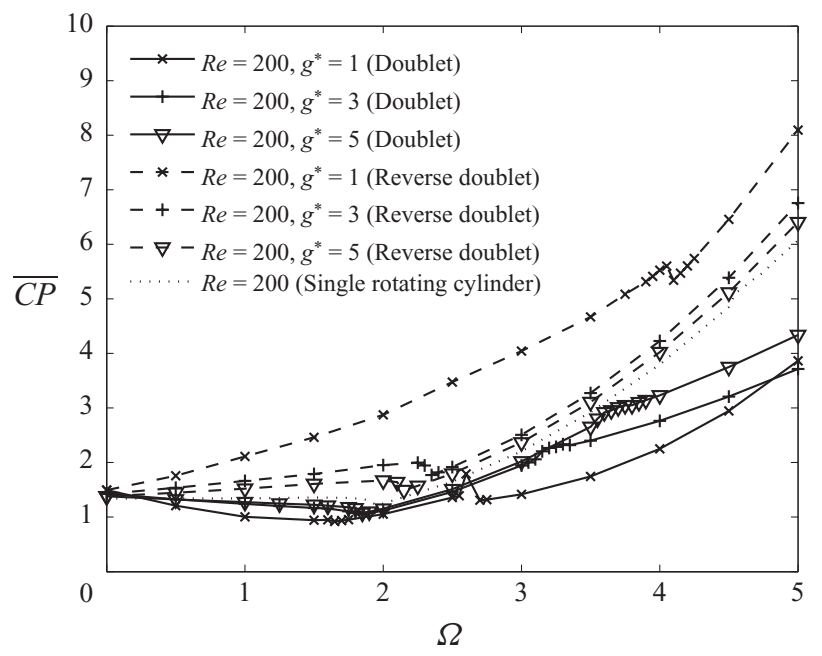

FIGURE 27. Flow past a counter-rotating cylinder: numerically determined time-averaged values of the power coefficient (upper cylinder, $R e=200$ ).
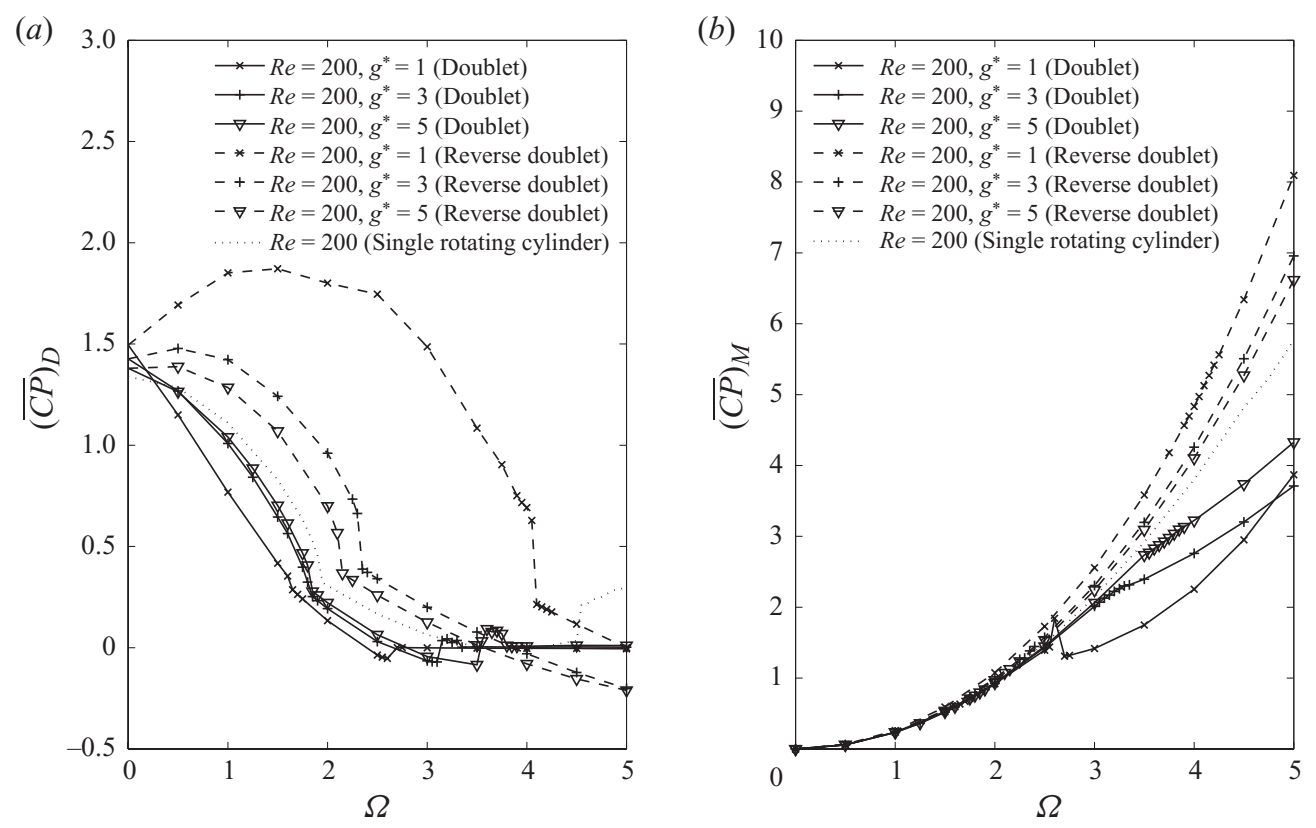

FIGURE 28. Flow past a counter-rotating cylinder: contributions of aerodynamic drag $(a)$ and spinning torque $(b)$ to the mean power coefficient (upper cylinder, $R e=200$ ).

In the reverse-doublet configuration, no change in the slope of $(\overline{C P})_{M}$ in figure $28(b)$ was observed at any gap spacing, since neither a closed body nor a secondary instability were present for the range rotational speeds examined here. Deviations in the slope of $\overline{C P}$ are the result of changes in the aerodynamic drag contribution, $(\overline{C P})_{D}$, and a sharp transition occurs during the drag reduction phase just prior to the total suppression of unsteady wakes at $\Omega=4.1,2.3$ and 2.25 for $g^{*}=1,3$ and 5, respectively (see figures 27 and 28). 


\section{Conclusions}

Digital particle image velocimetry and a high-order spectral difference computational method were employed to investigate the flow over a pair of counterrotating cylinders. The experimental and computational results were generally in excellent agreement in resolving the wake structure and various stability regimes.

For the doublet-like rotational configuration, a synchronized in-phase vortex shedding mode that was unstable to small disturbances was present at $R e=150$ and 200 for $g^{*}=1$. Decreasing the Reynolds number to 100 for this case resulted in a wake structure similar to that of flow past a single bluff body. The existence of a virtual elliptic body, first predicted by Chan \& Jameson (2010) to appear when the rotational speed is sufficiently large, was validated by the high-order SD computations, as well as by the experimental results. The size of the virtual elliptic body increases with increasing rotational speed. The values of the critical rotation rate $\Omega_{\text {crit }}$, where the wake forms an elliptic body and the total drag reduces to zero, were found more precisely than in this previous work, and over a wider range of Reynolds number and gap sizes. In addition, for the first time, a secondary instability was observed for $g^{*}=3$ and 5 , at rotational speeds just prior to the formation of the virtual elliptic body.

For the reverse doublet-like rotational configuration, the in-phase shedding mode was present for $g^{*}=1$ at all Reynolds numbers when $\Omega<1$, and the anti-phase shedding mode was present when $1<\Omega<\Omega_{\text {crit }}$. This is similar to that seen for a pair of non-rotating cylinders, but it was shown here for the first time that counter-rotation of the cylinder pair would lead to wake stabilization by transition to an anti-phase shedding mode for $1<\Omega<\Omega_{\text {crit }}$.

For both rotational configurations, a stable anti-phase wake structure was observed for all Reynolds numbers at $g^{*}=3$ and 5. For the doublet-like configuration, the value of $\Omega_{\text {crit }}$ monotonically increased with increasing gap spacing and Reynolds number, while for the reverse doublet-like configuration, it monotonically decreased with increasing gap spacing and Reynolds number. These opposing tendencies of the two rotational configurations with increasing gap spacing cause $\Omega_{\text {crit }}$ to converge towards the value needed to suppress vortex formation in the wake of a single rotating cylinder.

We would like to acknowledge K. Ou and S. Premasuthan for their help in verifying some of our findings and performing a comparative study using a commercial CFD code-STAR-CD. We would like to thank M. Pontecorvo and S. Bailey for designing and constructing the experimental apparatus. We are indebted to both the National Science Foundation and the Air Force Office of Scientific Research for their support of the research that led to this paper through grants 0708071 and FA9550-07-1-0195. The first author (A.S.C.) would especially like to acknowledge Hitachi Global Storage Technologies for supporting his PhD research. The third author (A.J.) is also grateful for the support of NASA Dryden Research Center through an STTR contract under the direction of Dr K. Gupta.

\section{Appendix. Validation results of the present numerical method}

A.1. Single stationary circular cylinder at $R e=100,150$ and 200

Here, we compare our SD numerical results for the flow past a single stationary circular cylinder at $R e=100,150$ and 200 with the results from a number of previous 


\begin{tabular}{|c|c|c|c|c|}
\hline \multicolumn{5}{|c|}{$R e=100$} \\
\hline Source & $S t$ & $C_{L}$ & $C_{D}$ & $\overline{C_{p b}}$ \\
\hline Present (SD, Fourth order) & 0.164 & \pm 0.325 & $1.338 \pm 0.009$ & -0.70 \\
\hline Present (Experiment) & 0.163 & - & - & - \\
\hline Braza, Chassaing \& Ha Minh (1986) & 0.160 & \pm 0.25 & $1.364 \pm 0.015$ & - \\
\hline Ding et al. (2007) & 0.166 & \pm 0.287 & $1.356 \pm 0.010$ & - \\
\hline Henderson (1994) & 0.165 & - & - & -0.73 \\
\hline Kovasznay (1949) & 0.163 & - & - & - \\
\hline Liu, Zheng \& Sung (1998) & 0.164 & \pm 0.339 & $1.350 \pm 0.012$ & - \\
\hline Park, Kwon \& Choi (1998) & 0.165 & \pm 0.332 & $1.33 \pm 0.009$ & -0.74 \\
\hline Sharman et al. (2005) & 0.164 & \pm 0.325 & $1.33 \pm 0.009$ & -0.72 \\
\hline Williamson $(1989,1996)$ & 0.164 & - & - & -0.71 \\
\hline \multicolumn{5}{|c|}{$R e=150$} \\
\hline Present (SD, Fourth order) & 0.184 & \pm 0.518 & $1.326 \pm 0.026$ & -0.84 \\
\hline Present (Experiment) & 0.184 & - & 1000 & - \\
\hline Belov, Martinelli \& Jameson (1995) & 0.182 & \pm 0.486 & $1.168 \pm 0.025$ & -0.82 \\
\hline Chan \& Jameson (2010) & 0.181 & \pm 0.531 & $1.331 \pm 0.028$ & -0.80 \\
\hline Henderson $(1994,1995)$ & 0.186 & - & - & -0.87 \\
\hline Kovasznay (1949) & 0.180 & - & - & - \\
\hline Park et al. (1998) & 0.184 & \pm 0.516 & $1.32 \pm 0.026$ & -0.85 \\
\hline Williamson $(1989,1996)$ & 0.184 & - & - & -0.85 \\
\hline \multicolumn{5}{|c|}{$R e=200$} \\
\hline Present (SD, Fourth order) & 0.196 & \pm 0.68 & $1.340 \pm 0.045$ & -0.96 \\
\hline Present (Experiment) & 0.197 & - & - & - \\
\hline Belov et al. (1995) & 0.193 & \pm 0.64 & $1.19 \pm 0.042$ & -0.94 \\
\hline Braza et al. (1986) & 0.200 & \pm 0.75 & $1.40 \pm 0.05$ & - \\
\hline Ding et al. (2007) & 0.196 & \pm 0.659 & $1.348 \pm 0.050$ & - \\
\hline Henderson (1994) & 0.197 & $-{ }_{-}$ & $\frac{-}{-}$ & -1.01 \\
\hline Kovasznay (1949) & 0.193 & - & - & - \\
\hline Lecointe \& Piquet (1984) & 0.194 & \pm 0.5 & $1.58 \pm 0.0035$ & - \\
\hline Liu et al. (1998) & 0.192 & \pm 0.69 & $1.31 \pm 0.049$ & - \\
\hline Mittal \& Kumar (2003) & 0.193 & \pm 0.66 & $1.316 \pm 0.049$ & - \\
\hline Rogers \& Kwak (1990) & 0.185 & \pm 0.65 & $1.23 \pm 0.05$ & - \\
\hline Miller \& Williamson (1994); Williamson (1996) & 0.197 & - & - & -0.86 \\
\hline
\end{tabular}

studies, as shown in table 2. The total number of cells used in this validation case is 6384. The grid distribution and boundary conditions are set in a similar and consistent manner as those used in the present research. The results were computed using the fourth-order SD refinement, with 102144 degrees of freedom (DOFs). The Strouhal numbers at $R e=100$ and 150 are identical to the experimental values reported by Williamson (1989). The computed mean base pressure coefficients, $\overline{C_{p b}}$, at these two Reynolds numbers are -0.70 and -0.84 , respectively, and they also are in excellent agreement with the experimental values reported by Williamson \& Roshko (1990). In addition, our computed lift and drag coefficients are well within the range of values reported in previous work. However, there appear to be some discrepancies at $R e=200$, which may be due to the presence of flow three-dimensionality which our two-dimensional code cannot resolve. Nonetheless, the Strouhal number agrees well with the computational value by Henderson (1994) and the upper transient value 


$\begin{array}{lcccrcc}\text { Mach number } & C_{D} & C_{D p} & C_{D v} & C_{L} & C_{L p} & C_{L v} \\ 0.05 & 0.0581 & -0.3481 & 0.4061 & 10.2450 & 9.8253 & 0.4193 \\ 0.1 & 0.0637 & -0.3353 & 0.3990 & 9.9640 & 9.5472 & 0.4168 \\ 0.2 & 0.0901 & -0.2747 & 0.3648 & 8.8534 & 8.4456 & 0.4078\end{array}$

TABLE 3. Flow past a single rotating circular cylinder: $C_{L}$ and $C_{D}$ variations as a function of Mach number at $R e=200, \omega=3 \Omega$ (steady flow). Subscripts: $p=$ pressure and $v=$ viscous.

from the hysteretic transition regime reported by Miller \& Williamson (1994) and Williamson (1996).

\section{A.2. Single rotating circular cylinder at $R e=200$}

Using the mesh from the stationary case, a non-slip, isothermal, moving boundary condition is applied at the rotating circular cylinder wall. The mean and standard deviation values of lift and drag, the Strouhal number and the coefficient of power are plotted in figure 29 as a function of rotational speeds for three different orders of accuracy. The figure also shows a comparison with the incompressible flow solutions using a finite-element formulation reported by Mittal \& Kumar (2003). In the lower speed range, only slight variations in the flow solutions are seen amongst the thirdand fourth-order SD methods and Mittal \& Kumar's method. The second-order SD method for the current mesh is not suitable for achieving the final flow solution. However, one of the advantages of our current numerical method is that a flow solution from the second order can be used as an initial condition for starting the more accurate higher order solution. Therefore, instead of using a higher order and letting the solution develop from an impulsive start, appreciable computation time can be saved with this restarting technique.

As the rotational speed increases, it is apparent that a larger discrepancy occurs between the solutions from our compressible SD code and those using Mittal \& Kumar's incompressible flow solver. Our solutions show the occurrence of the secondary instability in the speed range of $4.5<\Omega<5.15$ as compared to Mittal \& Kumar's solution of $4.4<\Omega<4.8$. One explanation for the difference is that the compressibility effect can no longer be neglected when the circular cylinder is rotated at a high enough speed. Figure 30 and table 3 show the effect of compressibility on density and Mach contours and on the force coefficients at $R e=200, \omega=3 \Omega$, calculated at free stream Mach numbers, $M a$, of $0.05,0.10$ and 0.20 . For $M a=0.2$, the local Mach number around the cylinder spinning at a very high rotational speed, e.g. $\Omega=5$, can become supersonic, thus yielding an entirely different flow problem. For very low Mach numbers, such as the case of $M a=0.05$, our solver suffers the same inefficiency as most other density-based compressible flow solvers. In this research, we use $M a=0.1$ since it is efficient, robust and yields results very close to those obtained using incompressible flow solvers, as shown in this section for the single rotating cylinder and in figure 10 for the counter-rotating cylinder pair.

\section{A.3. Study of mesh density and SD order of accuracy}

The numerical accuracy of different SD schemes has been documented by Ou et al. (2009). Here, an additional numerical accuracy test based on the mesh density and the SD order of accuracy is presented in table 4 for the three gap sizes considered in this research. Listed are the mean and standard deviation values of force coefficients and the corresponding Strouhal numbers (for the anti-phase shedding pattern) of the 

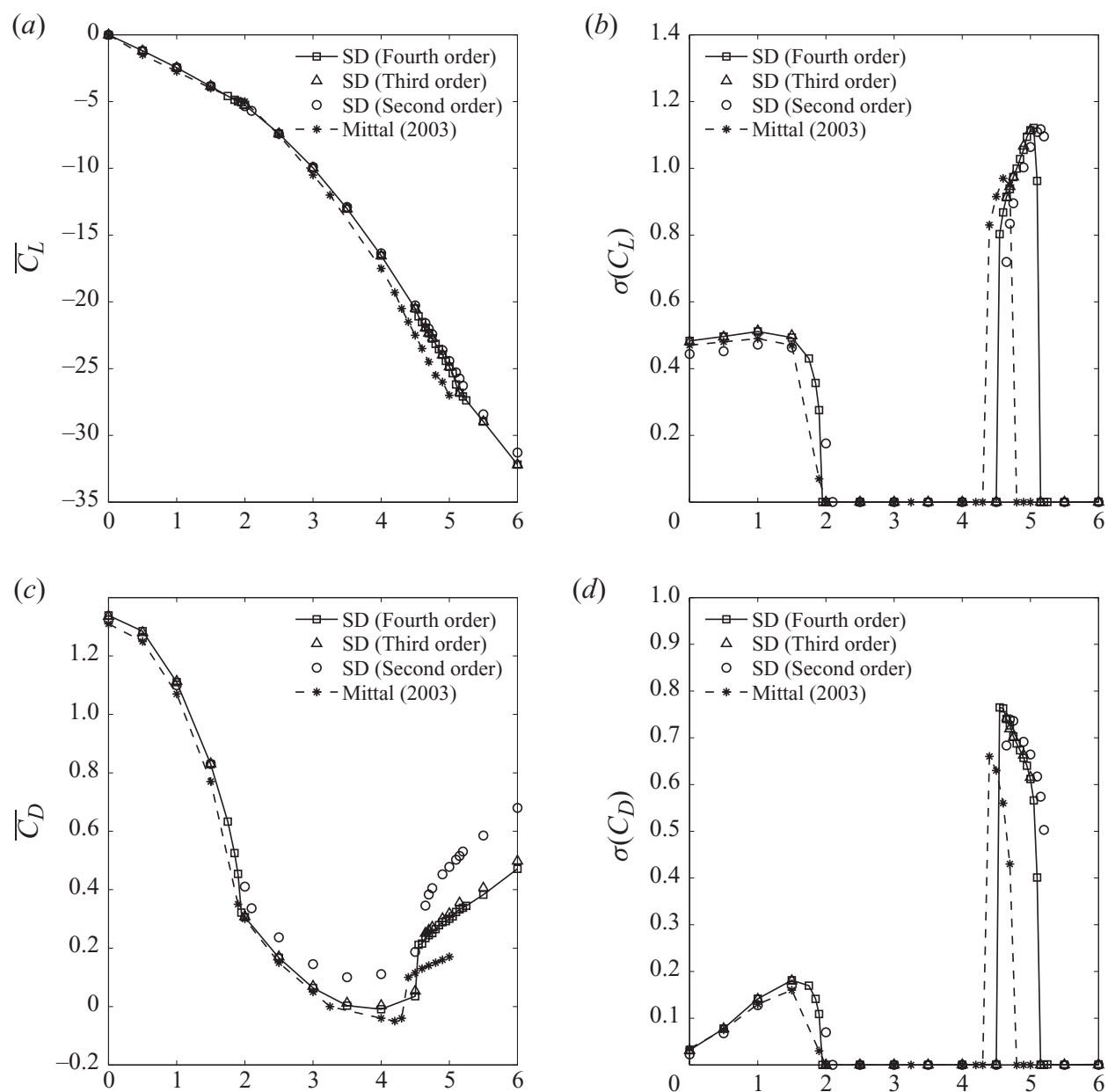

(d)
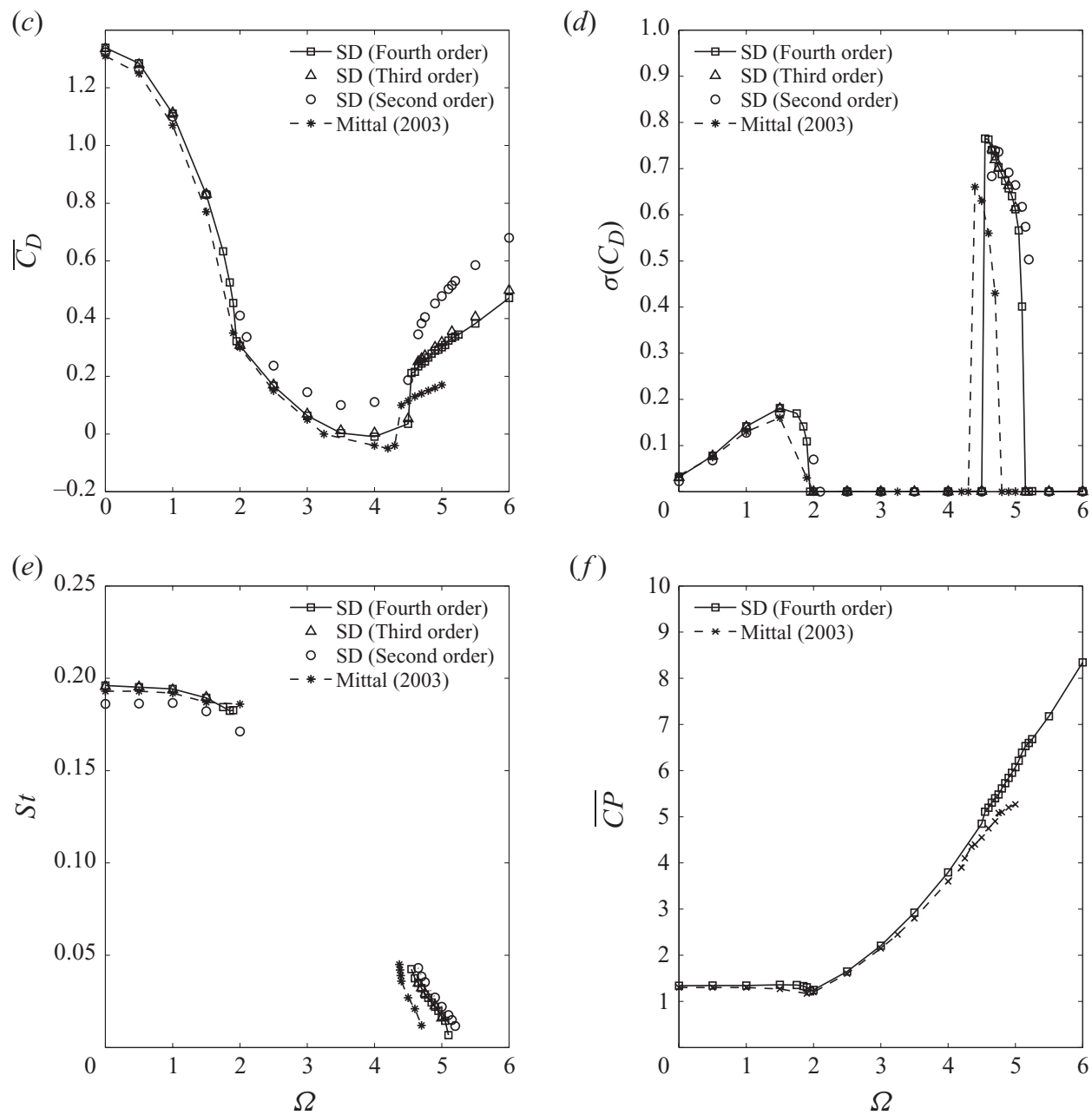

FiguRE 29. Flow past a rotating circular cylinder: mean $C_{L}(a)$, standard deviation of $C_{L}$ $(b)$, mean $C_{D}(c)$, standard deviation of $C_{D}(d), S t(e)$ and mean $C P(f)$. 


$\begin{array}{cccccccccccc}\text { Configuration } & R e & g^{*} & \Omega & \text { SD order } & \text { Number of cells } & \text { DOFs } & \overline{C_{D}} & \overline{C_{L}} & \sigma\left(C_{D}\right) & \sigma\left(C_{L}\right) & S t \\ 1 & 150 & 1 & 0 & 2 & 5176 \text { (Coarse) } & 20704 & 1.502 & 0.247 & 0.270 & 0.356 & - \\ & 150 & 1 & 0 & 2 & 20704 \text { (Fine) } & 82816 & 1.504 & 0.232 & 0.369 & 0.395 & - \\ & 150 & 1 & 0 & 4 & 5176 \text { (Coarse) } & 82816 & 1.474 & 0.227 & 0.110 & 0.388 & - \\ & 150 & 1 & 0 & 4 & 20704 \text { (Fine) } & 331264 & 1.472 & 0.228 & 0.107 & 0.361 & - \\ 2 & 150 & 1 & 3 & 2 & 5176 \text { (Coarse) } & 20704 & 0.082 & 6.045 & 0.000 & 0.000 & - \\ & 150 & 1 & 3 & 2 & 20704 \text { (Fine) } & 82816 & 0.028 & 6.067 & 0.000 & 0.000 & - \\ & 150 & 1 & 3 & 4 & 5176 \text { (Coarse) } & 82816 & 0.001 & 6.063 & 0.000 & 0.000 & - \\ & 150 & 1 & 3 & 4 & 20704 \text { (Fine) } & 331264 & 0.002 & 6.077 & 0.000 & 0.000 & - \\ 3 & 150 & 3 & 0 & 2 & 7736 \text { (Coarse) } & 30944 & 1.457 & 0.063 & 0.020 & 0.420 & 0.183 \\ & 150 & 3 & 0 & 2 & 30944 \text { (Fine) } & 123776 & 1.444 & 0.061 & 0.022 & 0.431 & 0.190 \\ & 150 & 3 & 0 & 4 & 7736 \text { (Coarse) } & 123776 & 1.421 & 0.057 & 0.023 & 0.429 & 0.193 \\ & 150 & 3 & 0 & 4 & 30944 \text { (Fine) } & 495104 & 1.422 & 0.058 & 0.023 & 0.429 & 0.192 \\ 4 & 150 & 3 & 3 & 2 & 7736 \text { (Coarse) } & 30944 & 0.060 & 9.250 & 0.000 & 0.000 & - \\ & 150 & 3 & 3 & 2 & 30944 \text { (Fine) } & 123776 & -0.013 & 9.373 & 0.000 & 0.000 & - \\ & 150 & 3 & 3 & 4 & 7736 \text { (Coarse) } & 123776 & -0.069 & 9.391 & 0.000 & 0.000 & - \\ & 150 & 3 & 3 & 4 & 30944 \text { (Fine) } & 495104 & -0.069 & 9.388 & 0.000 & 0.000 & - \\ 5 & 150 & 5 & 0 & 2 & 9088 \text { (Coarse) } & 36352 & 1.420 & 0.032 & 0.017 & 0.382 & 0.180 \\ & 150 & 5 & 0 & 2 & 36352 \text { (Fine) } & 145408 & 1.403 & 0.030 & 0.019 & 0.390 & 0.186 \\ & 150 & 5 & 0 & 4 & 9088 \text { (Coarse) } & 145408 & 1.373 & 0.028 & 0.020 & 0.386 & 0.189 \\ & 150 & 5 & 0 & 4 & 36,352 \text { (Fine) } & 581632 & 1.373 & 0.028 & 0.020 & 0.386 & 0.188 \\ 6 & 150 & 5 & 3 & 2 & 9088 \text { (Coarse) } & 36352 & 0.098 & 9.452 & 0.000 & 0.001 & - \\ & 150 & 5 & 3 & 2 & 36352 \text { (Fine) } & 145408 & 0.029 & 9.549 & 0.000 & 0.000 & - \\ & 150 & 5 & 3 & 4 & 9088 \text { (Coarse) } & 145408 & -0.040 & 9.569 & 0.000 & 0.000 & - \\ & 150 & 5 & 3 & 4 & 36352 \text { (Fine) } & 581632 & -0.040 & 9.564 & 0.000 & 0.000 & -\end{array}$

TABLE 4. Numerical accuracy test based on SD order and mesh density: values of the force coefficients and Strouhal numbers of the top cylinder. The standard deviation of the force coefficients are denoted by the symbol $\sigma$.

\begin{tabular}{llllc} 
& \multicolumn{4}{c}{$R e=100$} \\
\hline$g^{*}$ & $\overline{C_{D}}$ & $\overline{C_{L}}$ & $\sigma\left(C_{L}\right)$ & Source \\
1 & 1.427 & 0.259 & 0.147 & Present (SD, Fourth order) \\
& 1.44 & 0.28 & 0.17 & Kang (2003) \\
3 & 1.444 & 0.074 & 0.281 & Present (SD, Fourth order) \\
& 1.47 & 0.08 & 0.28 & Kang (2003)
\end{tabular}

TABLE 5. Stationary circular cylinder pair: mean $C_{D}$, mean $C_{L}$ and standard deviation of $C_{L}$.

top circular cylinder of the counter-rotating cylinder pair, for the stationary case and for the case with $\Omega=3$. In this test case, we use two different sets of meshes - coarse and fine. The fine mesh is essentially reconstructed from the coarse mesh by simply quadrupling the number of cells. The differences between the results obtained from the coarse mesh and those from the fine mesh are small when the fourth-order SD is used. However, the computational cost increases proportional to the number of DOFs. Therefore, in order to perform a fairly large simulation matrix in a reasonable amount of time, the coarse meshes in conjunction with the fourth-order SD were used to obtain the final results presented in this paper.

\section{A.4. Stationary pair of circular cylinders}

Here, we compare the force coefficients for a stationary circular cylinder pair obtained using the fourth-order SD calculations with the numerical results using the immersed boundary method by Kang (2003). Table 5 lists the values of mean $C_{L}$ and $C_{D}$ 
(a)
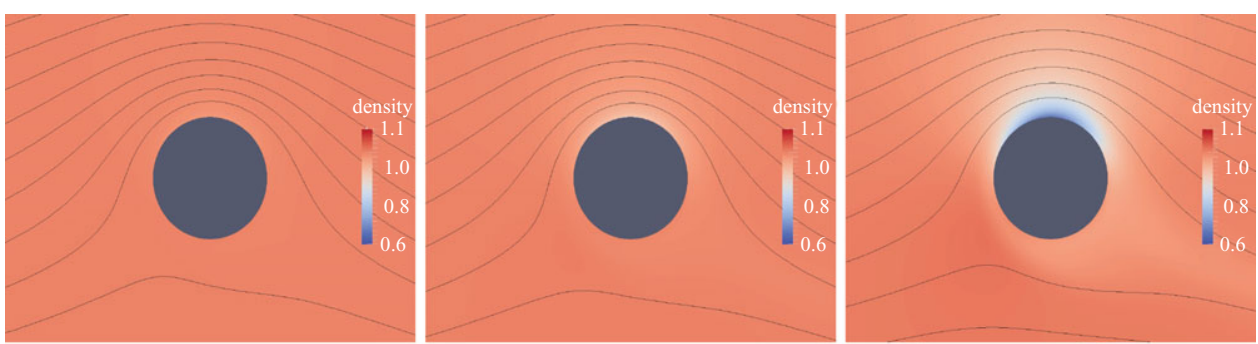

(b)
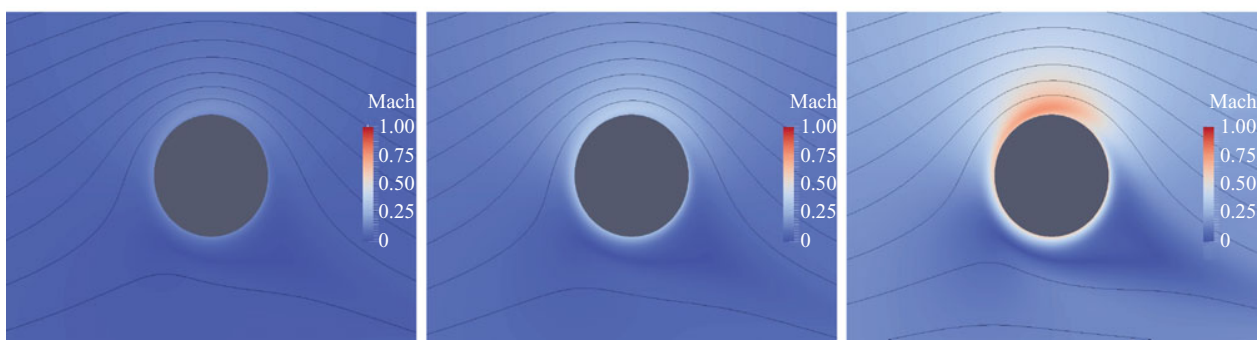

$M a=0.05$

$M a=0.1$

$M a=0.2$

FigURE 30. Flow past a single rotating circular cylinder, $R e=200, \omega=3 \Omega$ : normalized density $(a)$ and Mach $(b)$ contours at different free stream Mach numbers.

as well as the standard deviation of $C_{L}$ for the flip-flop shedding mode at $g^{*}=1$, $R e=100$ and for the anti-phase mode at $g^{*}=3, R e=100$. In general, the results are in reasonable agreement, further establishing our confidence in the approach used here.

\section{REFERENCES}

Belov, A., Martinelli, L. \& Jameson, A. 1995 A new implicit algorithm with multigrid for unsteady incompressible flow calculations. AIAA Paper 1995-0049, 33rd Aerospace Sciences Meeting and Exhibit, 9-12 January 1995, Reno, NV.

Braza, M., Chassaing, P. \& Ha Minh, H. 1986 Numerical study and physical analysis of the pressure and velocity fields in the near wake of a circular cylinder. J. Fluid Mech. 165, 79-130.

Chan, A. S. \& JAmeson, A. 2010 Suppression of the unsteady vortex wakes of a circular cylinder pair by a doublet-like counter-rotation. Intl J. Numer. Meth. Fluids 63, 22-39.

Cockburn, B., Hou, S. \& Shu, C. W. 1990 TVB Runge-Kutta local projection discontinuous Galerkin finite element method for conservation laws. Part IV: The multidimensional case. Math. Comput. 54, 545-581.

Cockburn, B., Lin, S. Y. \& ShU, C. W. 1989 TVB Runge-Kutta local projection discontinuous Galerkin finite element method for conservation laws. Part III: One-dimensional systems. $J$. Comput. Phys. 84, 90-113.

Cockburn, B. \& Shu, C. W. 1989 TVB Runge-Kutta local projection discontinuous Galerkin finite element method for conservation laws. Part II: General framework. Math. Comput. 52, $411-435$.

Cockburn, B. \& Shu, C. W. 1998 TVB Runge-Kutta local projection discontinuous Galerkin finite element method for conservation laws. Part V: Multidimensional system. J. Comput. Phys. 141, 199-224.

Ding, H., Shu, C., Yeo, K. S. \& Xu, D. 2007 Numerical simulation of flows around two circular cylinders by mesh-free least square-based finite difference methods. Intl J. Numer. Meth. Fluids 53, 305-332.

Harten, A. \& Hyman, J. M. 1983 Self-adjusting grid methods for one-dimensional hyperbolic conservation laws. J. Comput. Phys. 50, 235-269. 
Henderson, R. D. 1994 Unstructured spectral element methods: Parallel algorithms and simulations. PhD thesis, Princeton University.

Henderson, R. D. 1995 Details of the drag curve near the onset of vortex shedding. Phys. Fluids 7, 2102-2104.

Hesthaven, J. S. \& Warburton, T. 2008 Nodal Discontinuous Galerkin Methods - Algorithms, Analysis, and Applications. Springer.

HuYnh, H. T. 2007 A flux reconstruction approach to high-order schemes including discontinuous Galerkin methods. AIAA Paper 2007-4079, 18th AIAA CFD Conference, 25-28 June 2007, Miami, FL.

JAMESON, A. 2010 A proof of the stability of the spectral difference method for all orders of accuracy. J. Sci. Comput. 45, 348-358

JiMÉNEZ, J. M. 2002 Low Reynolds number studies in the wake of a submarine model using particle image velocimetry. MSE thesis, Princeton University.

KANG, S. 2003 Characteristics of flow over two circular cylinders in a side-by-side arrangement at low Reynolds numbers. Phys. Fluids 15, 2486-2498.

Kang, S., ChOI, H. \& LeE, S. 1999 Laminar flow past a rotating circular cylinder. Phys. Fluids 11, 3312-3321.

Kim, H. J. \& Durbin, P. A. 1988 Investigation of the flow between a pair of circular cylinders in the flopping regime. J. Fluid Mech. 196, 431-448.

Kopriva, D. A. \& Kolias, J. H. 1996 A conservative staggered-grid Chebyshev multidomain method for compressible flows. J. Comput. Phys. 125 (1), 244-261.

KovasznaY, L. S. G. 1949 Hot-wire investigation of the wake behind cylinders at low Reynolds numbers. Proc. R. Soc. Lond. A 198, 174-190.

LeCoINTE, Y. \& PiQuet, J. 1984 On the use of several compact methods for the study of unsteady incompressible viscous flow round a circular cylinder. Comput Fluids 12, 255-280.

LiAng, C., JAMEson, A. \& WANG, Z. J. 2009 a Spectral difference method for two-dimensional compressible flow on unstructured grids with mixed elements. J. Comput. Phys. 228, 28472858.

Liang, C., Premasuthan, S. \& Jameson, A. $2009 \mathrm{~b}$ High-order accurate simulation of flow past two side-by-side cylinders with spectral difference method. J. Comput. Struct. 87, 812-817.

Liang, C., Premasuthan, S., Jameson, A. \& Wang, Z. J. 2009c Large eddy simulation of compressible turbulent channel flow with spectral difference method. AIAA Paper 2009-402, 47th AIAA Aerospace Sciences Meeting, 5-8 January 2009, Orlando, FL.

Liu, C., Zheng, X. \& Sung, C. H. 1998 Preconditioned multigrid methods for unsteady incompressible flows. J. Comput. Phys. 139, 35-57.

LiU, Y., VinOKuR, M. \& WANG, Z. J. 2006 Spectral difference method for unstructured grids. Part I: Basic formulation. J. Comput. Phys. 216, 780-801.

Miller, G. D. \& Williamson, C. H. K. 1994 Control of three-dimensional phase dynamics in a cylinder wake. Exp. Fluids 18, 26-35.

Mittal, S. \& Kumar, B. 2003 Flow past a rotating cylinder. J. Fluid Mech. 476, 303-334.

Mohammad, A. H., Wang, Z. J. \& Liang, C. 2008 LES of turbulent flow past a cylinder using spectral difference method. AIAA Paper 2008-7184. 26th AIAA Applied Aerodynamics Meeting, 18-21 August 2008, Honolulu, HI.

Ou, K., Liang, C., Premasuthan, S. \& Jameson, A. 2009 High-order spectral difference simulation of laminar compressible flow over two counter-rotating cylinders. AIAA Paper 2009-3956, 27th AIAA Applied Aerodynamics Conference, 22-25 June 2009, San Antonio, TX

Park, J., Kwon, K. \& CHOI, H. 1998 Numerical solutions of flow past a circular cylinder at Reynolds numbers up to 160. KSME Intl J. 12, 1200-1205.

Peschard, I. \& Le Gal, P. 1996 Coupled wakes of cylinders. Phys. Rev. Lett. 77, 3122-3125.

Prandtl, L. \& Tietsens, O. G. 1934 Applied Hydro- and Aeromechanics, Dover edn. 1957. Dover Publications.

Premasuthan, S., Liang, C., Jameson, A. \& Wang, Z. J. 2009 A P-multigrid spectral difference method for viscous flow. AIAA Paper 2009-950, 47th AIAA Aerospace Sciences Meeting, 5-8 January 2009, Orlando, FL.

Raffel, M., Willert, C., Wereley, S. \& Kompenhans, J. 1998 Particle Image Velocimetry. Springer.

RoE, P. L. 1981 Approximate Riemann solvers, parameter vectors, and difference schemes. J. Comput. Phys. 43, 357-372. 
Rogers, S. E. \& KwaK, D. 1990 Upwind differencing scheme for the time-accurate incompressible Navier-Stokes equations. AIAA J. 28, 253-262.

Sharman, B., Lien, F. S., Davidson, L. \& Norberg, C. 2005 Numerical predictions of low Reynolds number flows over two tandem circular cylinders. Intl J. Numer. Meth. Fluids 47, 423-447.

SPItERI, R. J. \& RUUTH, S. J. 2002 A new class of optimal high-order strong-stability-preserving time discretization methods. SIAM J. Numer. Anal. 40, 469-491.

Sumner, D., Wong, S. S. T., Price, S. J. \& Paidoussis, M. P. 1999 Fluid behaviour of side-by-side circular cylinders in steady cross-flow. J. Fluids Struct. 13, 309-338.

WANG, Z. J., LiU, Y., MaY, G. \& JAMESON, A. 2007 Spectral difference method for unstructured grids. Part II: Extension to the Euler equations. J. Sci. Comput. 32 (1), 45-71.

Whittlesey, R. W., Liska, S. \& DabiRi, J. O. 2010 Fish schooling as a basis for vertical axis wind turbine farm design Bioinsp. Biomim. 5, 035005(1-6).

Williamson, C. H. K. 1985 Evolution of a single wake behind a pair of bluff bodies. J. Fluid Mech. 159, $1-18$.

Williamson, C. H. K. 1989 Oblique and parallel modes of vortex shedding in the wake of a circular cylinder at low Reynolds numbers. J. Fluid Mech. 206, 579-627.

Williamson, C. H. K. \& Roshko, A. 1990 Measurements of base pressure in the wake of a cylinder at low Reynolds numbers. Z. Flugwiss. Weltraumforsch 14, 38-46.

Williamson, C. H. K. 1996 Vortex dynamics in the cylinder wake. Annu. Rev. Fluid Mech. 28, $477-539$.

Xu, S. J., Zhou, Y. \& So, R. M. C. 2003 Reynolds number effects on the flow structure behind two side-by-side cylinders. Phys. Fluids 15, 1214.

Yoon, H. S., Kim, J. H., Chun, H. H. \& ChOI, H. J. 2007 Laminar flow past two rotating cylinders in a side-by-side arrangement. Phys. Fluids 19, 128103-1-128103-4. 